This is the post peer-review accepted manuscript of:

M. Chiani, A. Giorgetti and E. Paolini, "Sensor Radar for Object Tracking," in Proceedings of the IEEE, vol. 106, no. 6, pp. 1022-1041, June 2018.

The published version is available online at:

https://doi.org/10.1109/JPROC.2018.2819697

C 2018 IEEE. Personal use of this material is permitted. Permission from IEEE must be obtained for all other uses, in any current or future media, including reprinting/republishing this material for advertising or promotional purposes, creating new collective works, for resale or redistribution to servers or lists, or reuse of any copyrighted component of this work in other works. 


\title{
Sensor Radar for Object Tracking
}

\author{
Marco Chiani, Fellow, IEEE, Andrea Giorgetti, Senior Member, IEEE, and Enrico Paolini, Member, IEEE
}

(Invited Paper)

\begin{abstract}
Precise localization and tracking of moving objects is of great interest for a variety of emerging applications including the Internet-of-Things (IoT). The localization and tracking tasks are challenging in harsh wireless environments, such as indoor ones, especially when objects are not equipped with dedicated tags (non-collaborative). The problem of detecting, localizing, and tracking non-collaborative objects within a limited area has often been undertaken by exploiting a network of radio sensors, scanning the zone of interest through wideband radio signals to create a radio image of the objects. This paper presents a sensor network for radio imaging (sensor radar) along with all of the signal processing steps necessary to achieve highaccuracy objects tracking in harsh propagation environment. The described sensor radar is based on the impulse radio (IR) ultrawide-band (UWB) technology, entailing the transmission of very short duration pulses. Experimental results with actual UWB signals in indoor environments confirm the sensor radar's potential in IoT applications.
\end{abstract}

Index Terms-Detection, indoor tracking, Internet-of-Things, localization, multistatic radar, sensor radar, ultrawide-band.

\section{INTRODUCTION}

$\mathbf{L}$ OCALIZATION and tracking of moving objects is a critical component for important new applications, including medical services, military systems, search and rescue operations, automotive safety, and logistics [1]-[7]. Indeed, wireless sensor networks and more generally the Internet-ofThings (IoT) consider localization as the enabling technology to provide georeferenced information from sensors, machines, vehicles, and wearable devices, in the ever growing trend of hyper-connected society [8]-[11].

Localization systems estimate object positions based on observations (or measurements) gathered by a network of sensors deployed in the environment [12]-[17]. The term "localization" usually refers to the capability to infer the position of collaborative objects, i.e., tagged objects interacting with the system to facilitate the localization process [18]-[21]. However, an increasing attention is recently being devoted to the capability of detecting and tracking objects that do not take actions to help the localization infrastructure or that do not wish to be detected and localized at all. This operation is referred to non-collaborative localization and is typical of radar systems [22]. These systems are further classified based on whether the network emits a signal designed for target detection and localization (active radar), or the network exploits

This work was supported in part by the European Project EuroCPS through the H2020 Framework under Grant 644090.

The authors are with the Department of Electrical, Electronic, and Information Engineering "Guglielmo Marconi” (DEI), University of Bologna, via Venezia 52, 47521 Cesena (FC), ITALY e-mail: (\{marco.chiani, andrea.giorgetti, e.paolini\}@unibo.it). Author names are listed in alphabetical order. signals emitted by other sources of opportunity (passive radar) [23]-[25].

The expressions "sensor radar" and "radar sensor network" are hereafter used interchangeably to refer to a network of active radars in a monostatic, bistatic, or multistatic configuration [26]-[30]. A multistatic radar is a network of multiple, independent radars, where each sensor performs a significant amount of processing. Pre-processed data are then collected by central unit through a communication link [27]. Multistatic radar shall not be confused with multiple-input multiple-output (MIMO) radar, which refers to a radar system employing multiple transmit waveforms (typically orthogonal to each other) and having the ability to jointly process signals received at multiple antennas [31], [32]. Such a feature requires tight synchronization among all sensors, to track phase changes, and limits the possibility to perform local processing [33].

Accurate localization via sensor radars becomes particularly challenging in indoor environments characterized by dense multipath, clutter, signal obstructions (e.g., due to the presence of walls), and interference. In a real-world scenario measurements are usually heavily affected by such impairments, severely affecting detection reliability and localization accuracy. These operating conditions may be mitigated by the adoption of waveforms characterized by large bandwidth, e.g., ultrawide-band (UWB) ones, exploiting prior knowledge of the environment, selecting reliable measurements, and using a wide range of signal processing tools [34]-[37].

The UWB technology, and in particular its impulse radio (IR) version characterized by the transmission of a few nanoseconds duration pulses [38]-[45], offers an extraordinary resolution and localization precision in harsh environments, due to its ability to resolve multipath and penetrate obstacles [46]-[49]. Additional advantages include low power consumption, low probability of intercept, robustness to jamming, and coexistence with a large number of systems in the ever increasing spectrum exploitation [50]-[52]. These features, together with the property of IR-UWB devices to be light-weight, cost-effective, and characterized by low power emissions, have contributed to make UWB an ideal candidate for noncollaborative object detection in short-range radar sensor network (RSN) applications [53]-[65]. UWB has been employed in through wall imaging, which has the ability to locate indoor moving targets with a radar situated at a standoff range outside buildings [66]-[73]. It has also been successfully adopted in a number of related applications including, e.g., radio-frequency identification [74]-[76], search and rescue of trapped victims [77]-[81], vital sign detection and estimation [82], [83], stroke detection [84], people counting [85], environmental imaging [86], [87], and non-destructive testing [88]. In the sensor radar field a number of papers have addressed several aspects related 
TABLE I

LIST OF ACRONYMS

\begin{tabular}{ll}
\hline AWGN & additive white Gaussian noise \\
CA-CFAR & cell averaging CFAR \\
c.d.f. & cumulative distribution function \\
CFAR & constant false alarm rate \\
CPHD & cardinalized PHD \\
DP & direct path \\
DTB-CFAR & double threshold and buffer CFAR \\
EM & expectation-maximization \\
ER & empty-room \\
G-CFAR & global CFAR \\
IoT & Internet-of-Things \\
IR & impulse radio \\
LOS & line-of-sight \\
LS & least square \\
MHT & multiple hypothesis tracking \\
MIMO & multiple-input multiple-output \\
MTI & moving target indicator \\
p.d.f. & probability density function \\
PHD & probability hypothesis density \\
PSD & power spectral density \\
RCS & radar cross section \\
RE & residual error \\
RFS & random finite set \\
RMSE & root mean-square error \\
RSN & radar sensor network \\
Rx & receiver \\
SNR & signal-to-noise ratio \\
SUT & sample under test \\
ToA & time-of-arrival \\
Tx & transmitter \\
UWB & ultrawide-band \\
& \\
\hline &
\end{tabular}

to the use of UWB, e.g., clutter removal and channel modeling [89], [90], detection [91]-[95], target recognition [96], tracking [97]-[111], sensors deployment [57], [112], [113], sensors power allocation [114], and cognitive mechanisms [115][118].

The localization of non-collaborative objects poses several problems at different levels of the processing chain, ranging from clutter removal, ghost mitigation, detection and clustering, within each sensor, to measurement combining (data fusion), clustering, measurement selection and tracking, at the network level. Finding a way to carry out all of these processing steps and to exploit their mutual relationships is a hard task and requires an almost holistic view of the system. For this reason, this paper aims at presenting the main aspects that involve detection, localization and tracking of noncollaborative objects in indoor environments using off-theshelf IR-UWB technology. The most challenging problems at different system levels are presented and possible solutions are proposed. The presentation is kept at introductory level, providing in-depth references for the interested reader. A sensor radar demonstrator is used to address step-by-step processing of actual signals and data, which have been collected in a real indoor environment through an experimental campaign. The use of real equipments is important to gain insights from real-world waveforms and the associated challenging practical aspects. The paper describes all steps from actual raw signal acquisition to object tracking through the complex processing chain of the network. The localization error performance will confirm the effectiveness of the performed processing.

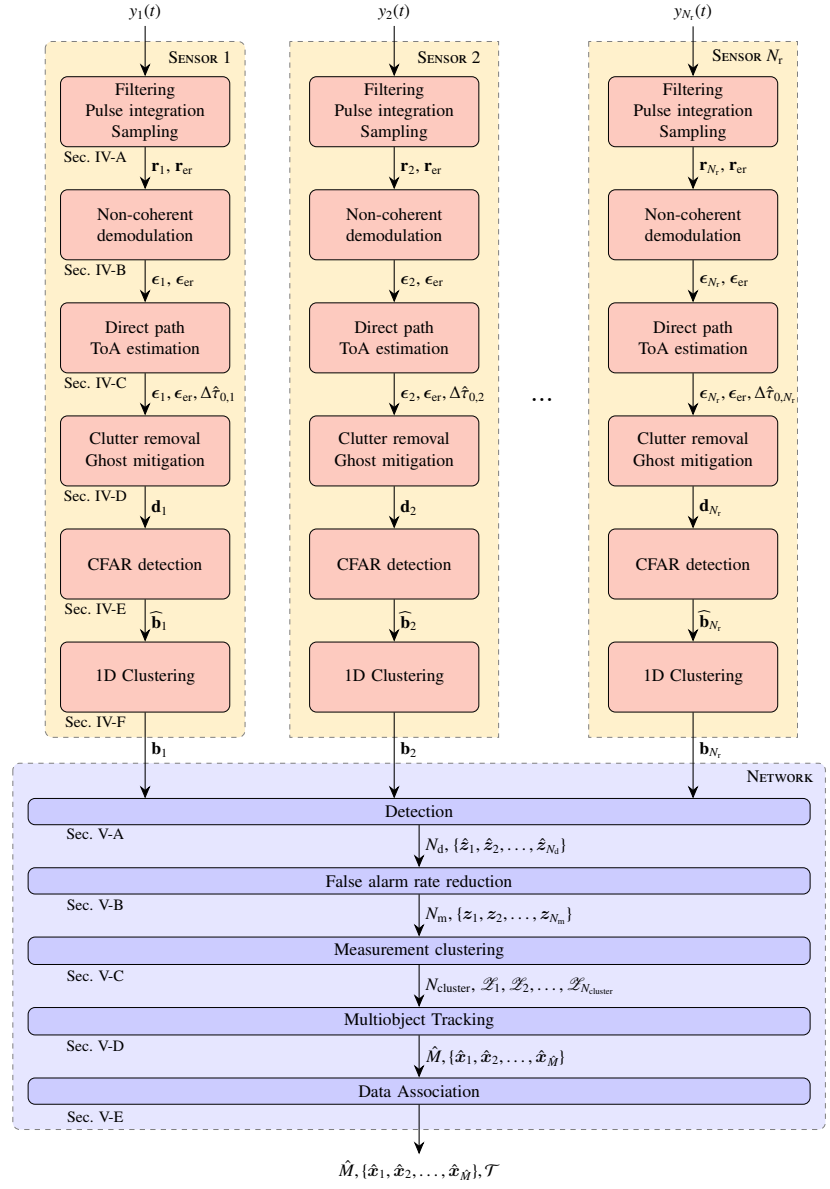

Fig. 1. Block diagram illustrating the processing performed by the RSN.

The paper is organized as follows. A system overview is provided in Sec. II and basic equations addressing system coverage are presented in Sec. III. Waveform processing within each sensor is detailed and illustrated in Sec. IV, and all processing steps for data fusion are presented in Sec. V. A case study analysis for human being tracking is provided in Sec. VI. Conclusions are drawn in Sec. VII. A list of frequently used abbreviations is provided in Table I.

\section{SySTEM OVERVIEW}

\section{A. Multistatic radar sensor networks}

We consider a RSN based on a UWB multistatic radar system. The RSN is aimed at detecting, locating, and tracking moving objects (also called targets in the radar jargon) within a given monitored area. The network is composed of one transmitter (Tx), $N_{\mathrm{r}}$ receivers (Rxs) or sensors, and one central node. Each Tx-Rx pair forms a monostatic or a bistatic radar depending on whether or not the transmitter and the receiver are co-located. In order for the RSN to be able to locate a target in the monitored area, the number of sensors shall fulfill $N_{\mathrm{r}} \geq 3$ in two dimensions. In each bistatic pair, a single signal transmission causes the reception of at least two signal replicas in free-space propagation: the direct signal via the Tx-to- $R x$ path and the reflected signal via the Tx-to-target-to-Rx path.

Fig. 1 shows a block diagram which summarizes the whole processing performed by the RSN in each frame (i.e., scan pe- 


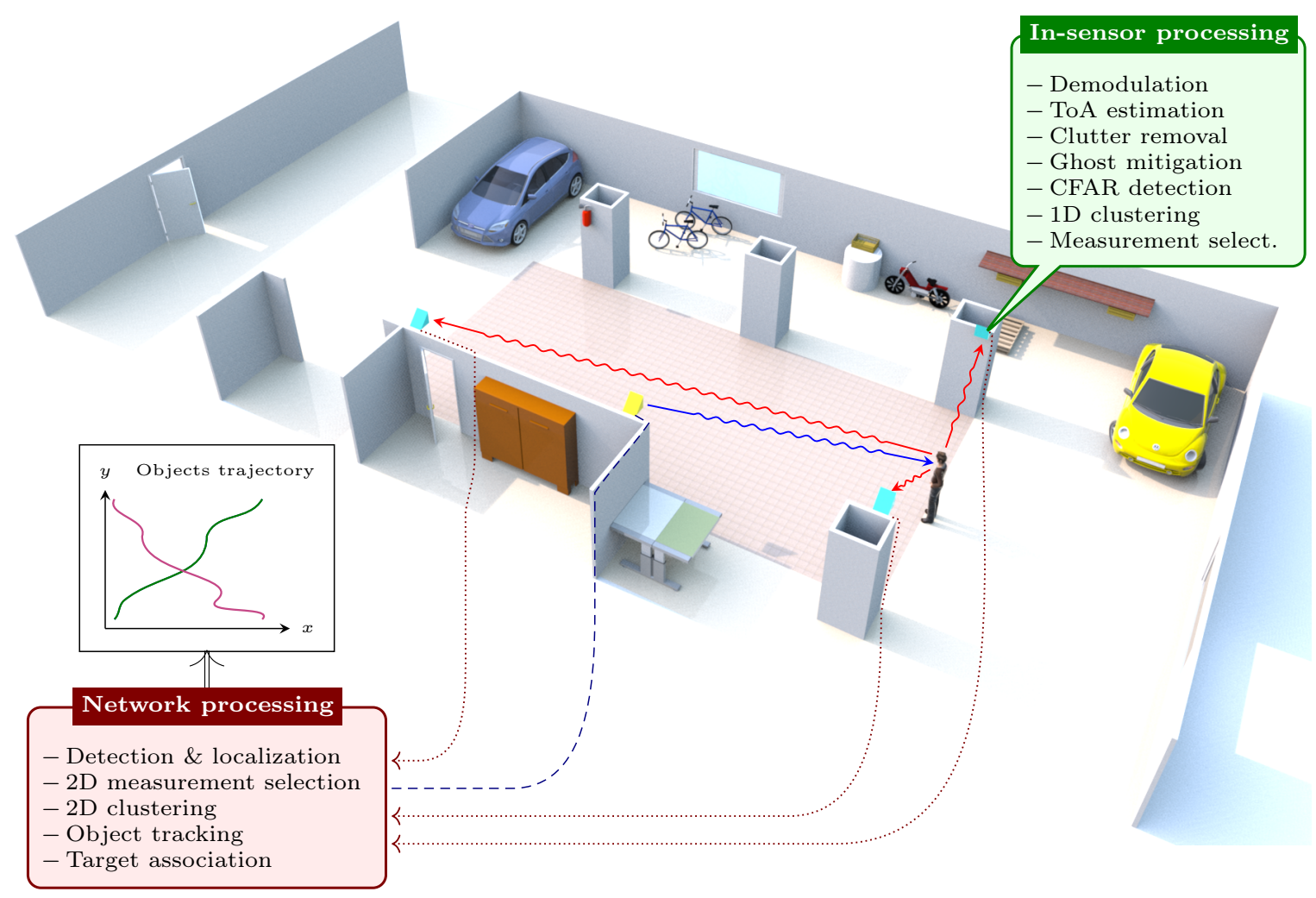

Fig. 2. A typical scenario for object tracking through a multistatic radar. The overall processing chain is divided into in-sensor processing and network processing for data fusion,

riod). There are $N_{\mathrm{r}}$ yellow boxes, each representing a specific sensor. The light red blocks in each "Sensor" box show all processing steps that are performed individually by each Rx. The light blue box labeled as "Network" represents the central node, which fuses information from all sensors to perform target detection and tracking; blocks included in it overview all processing steps performed at central level. Every block in Fig. 1 has one input and one output arrow, labeled with the data before and after its processing, respectively. Moreover, blocks are labeled with the number of the subsection in which the corresponding processing is described. In each frame, the RSN input is represented by the $N_{\mathrm{r}}$ received signals $y_{1}(t), y_{2}(t)$, $\ldots, y_{N_{\mathrm{r}}}(t)$, one per sensor, while its output is the current estimate $\hat{M}$ of the number of targets, the set $\left\{\hat{\boldsymbol{x}}_{1}, \hat{\boldsymbol{x}}_{2}, \ldots, \hat{\boldsymbol{x}}_{\hat{M}}\right\}$ of current target state estimates, and the set $\mathcal{T}$ of current tracks.

A pictorial representation of a typical RSN application scenario is provided in Fig. 2. Radio pulses generated by the Tx node are scattered by a human target and their echoes are received by $N_{\mathrm{r}}$ sensors $\left(N_{\mathrm{r}}=3\right.$ in the example). Similarly to Fig. 1, also Fig. 2 summarizes the processing steps that are carried out by each sensor in each scan period, as well as the ones carried out at network level.

\section{B. The radar waveform}

The transmitted signal is composed by a sequence of UWB pulses organized in frames. A frame is a sequence of $N_{\mathrm{s}}$ pulses at intervals $T_{\mathrm{IP}}$ (the inter-pulse period), therefore its duration is $T_{\mathrm{f}}=T_{\mathrm{IP}} N_{\mathrm{s}}$. The pulse is usually very short, with a duration of the order of a nanosecond. Several possible choices of the transmitted pulse have been proposed. Most of them relies on the first (or a higher order) derivative of a Gaussian monocycle [47], [119]. Due to the processing described in Sec. IV-A, $N_{\mathrm{s}}$ is called pulse integration factor.

The system is designed in such a way that the channel response to a single pulse when a moving target is present does not change appreciably during a frame time. ${ }^{1}$ If a target is present inside the area, the received signal at each Rx node corresponding to a transmitted pulse consists of the direct path pulse followed by pulse replicas, due to both the clutter and the target, and the additive white Gaussian noise (AWGN) [91].

\section{Area Coverage}

Let us consider a bistatic pair composed of one Tx and one $\mathrm{Rx}$ and let us denote by $\ell$ the distance between the two radio nodes (baseline). Moreover, let us denote by $\ell_{t}$ and $\ell_{r}$ the distances between the Tx and the target and the Rx and the target, respectively. Assuming for the moment freespace propagation, the received energy per pulse due to target scattering of the generic pulse emitted by the Tx, conditional on a specific (frequency dependent) realization $\sigma(f)$ of the

\footnotetext{
${ }^{1}$ This condition can be guaranteed by a proper parameter setting as detailed in Sec. IV-A.
} 
target radar cross section (RCS), may be expressed as

$$
E=\int_{f_{\mathrm{L}}}^{f_{\mathrm{L}}+B} \frac{\mathcal{E}(f) G_{t}(f) G_{r}(f) \sigma(f)}{(4 \pi)^{3}\left(\ell_{t} \ell_{r}\right)^{2}}\left(\frac{c}{f}\right)^{2} \mathrm{~d} f .
$$

In (1), $\mathcal{E}(f)$ is the energy spectral density of the transmitted pulse, $G_{t}(f), G_{r}(f)$ are the (frequency dependent) antenna gains, $\left(f_{\mathrm{L}}, f_{\mathrm{U}}\right)$ is the signal band, $B=f_{\mathrm{U}}-f_{\mathrm{L}}$ is the signal bandwidth, and $c$ is the speed of light. ${ }^{2}$

The signal-to-noise ratio (SNR) is defined as

$$
\mathrm{SNR}=\frac{E N_{\mathrm{s}}}{N_{0}}=\frac{\Gamma}{\left(\ell_{t} \ell_{r}\right)^{2}}
$$

where $N_{0}$ is the one-sided noise PSD and

$$
\Gamma=N_{\mathrm{s}} \int_{f_{\mathrm{L}}}^{f_{\mathrm{L}}+B} \frac{\mathcal{E}(f) G_{t}(f) G_{r}(f) \sigma(f)}{(4 \pi)^{3} N_{0}}\left(\frac{c}{f}\right)^{2} \mathrm{~d} f
$$

is often referred to as the bistatic radar constant. ${ }^{3}$ Denoting by $\mathrm{SNR}_{\min }$ the minimum SNR necessary to achieve required missed detection and false alarm probabilities, the largest value of the product $\ell_{t} \ell_{r}$ to meet the desired performance is given by

$$
\left(\ell_{t} \ell_{r}\right)_{\max }=\sqrt{\frac{\Gamma}{\mathrm{SNR}_{\min }}}
$$

and is often referred to as the bistatic range of the Tx-Rx pair.

From (2), over any plane containing both the Tx and the $\mathrm{Rx}$, the geometric curve corresponding to a constant product $\ell_{t} \ell_{r}$ may be thought as associated with a constant value of the SNR. ${ }^{4}$ Geometrically, such a curve is named a Cassini oval and is defined as the locus of the points in a plane such that the product of their distances from two fixed points, called foci, is a constant. The distance between the foci is usually denoted by $2 a$ and the constant by $b^{2}$. For a bistatic pair, the positions of the two foci coincide with the Tx and Rx ones, hence we have $2 a=\ell$ (baseline) and $b^{2}=\ell_{t} \ell_{r}$. The shape of a Cassini oval depends on the ratio

$$
\frac{b}{a}=\frac{\sqrt{\ell_{t} \ell_{r}}}{\ell / 2} .
$$

In particular, the curve consists of two loops around the foci when $\sqrt{\ell_{t} \ell_{r}}<\ell / 2$, it assumes a lemniscate shape when $\sqrt{\ell_{t} \ell_{r}}=\ell / 2$, and it is a single loop with a "dog bone" or an oval shape when $\sqrt{\ell_{t} \ell_{r}}>\ell / 2$. Examples of Cassini ovals are depicted in Fig. 3, where both axes are normalized with respect to $a=\ell / 2$. Using (2) and letting $\Gamma=5 \ell^{4}$ (arbitrarily), the

\footnotetext{
${ }^{2}$ An example of measured power spectral density (PSD), $S_{\mathrm{t}}(f)$, of the UWB apparatus adopted in our test-bed (see Sec. VI) can be found in [120]. An experimental investigation of the RCS, $\sigma(f)$, of the human body can be found in [121], [122].

${ }^{3} \mathrm{~A}$ related definition is the ratio $P_{\mathrm{r}} /\left(N_{0} B_{\text {eq }}\right)$ of the peak received target-scattered power to the average noise power ( $B_{\text {eq }}$ is the equivalent noise bandwidth of the pre-detection Rx filter). We have $P_{\mathrm{r}} /\left(N_{0} B_{\text {eq }}\right)=$ $\left(E / N_{0}\right) \mathrm{PRF} /\left(B_{\text {eq }} \mathrm{d}\right)$ where PRF is the pulse repetition frequency and $\mathrm{d}$ is the duty cycle (ratio of the pulse duration to $T_{\mathrm{IP}}$ ).

${ }^{4} \mathrm{~A}$ constant SNR over the curve assumes availability of a line-of-sight (LOS) radio path between both the Tx and the target and the Rx and the target, as well as invariant RCS and antenna gains. Although this may not be the case in reality, the model remains useful to introduce the concept of coverage.
}

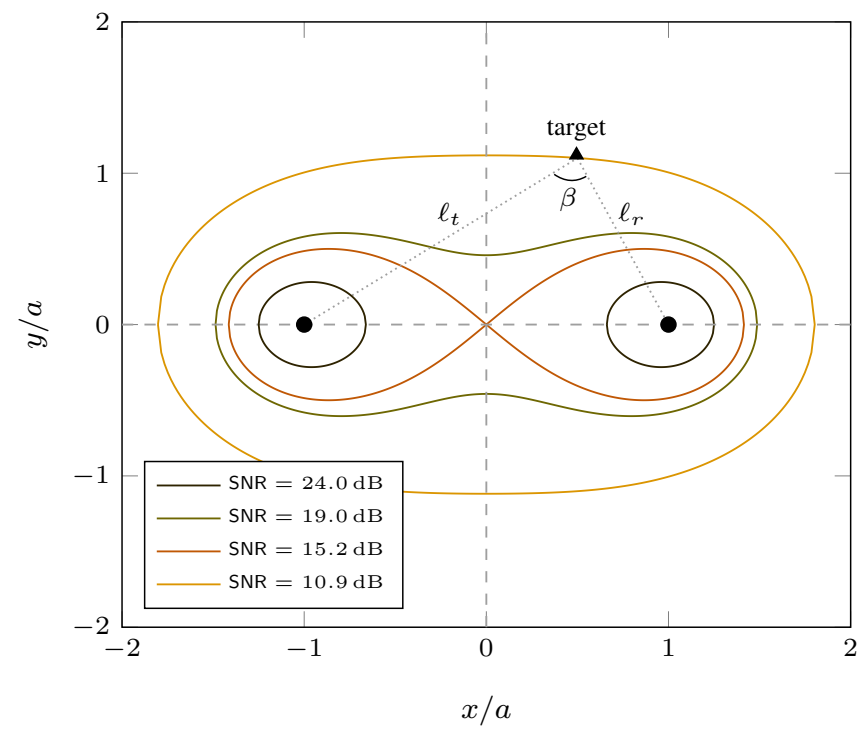

Fig. 3. Examples of Cassini ovals (iso-SNR curves for a bistatic pair). Both the $x$ - and the $y$-axis are normalized with respect to $a=\ell / 2$. The Tx and the $\mathrm{Rx}$ are in positions $(-\ell / 2,0)$ and $(\ell / 2,0)$. From the innermost curve to the outermost one: $\sqrt{\ell_{t} \ell_{r}} /(\ell / 2)=0.75$ (two loops), $\sqrt{\ell_{t} \ell_{r}} /(\ell / 2)=1$ (lemniscate), $\sqrt{\ell_{t} \ell_{r}} /(\ell / 2)=1.25$ ("dog bone"), $\sqrt{\ell_{t} \ell_{r}} /(\ell / 2)=1.6$ (oval).

innermost Cassini oval in the figure (two loops) is associated with $\mathrm{SNR}=24.0 \mathrm{~dB}$ while the outermost one (single oval) with $\mathrm{SNR}=10.9 \mathrm{~dB}$.

By definition, the maximum Cassini oval for a bistatic pair is the Cassini oval whose points are associated with $\mathrm{SNR}=\mathrm{SNR}_{\min }$. Equivalently, it is the Cassini oval whose parameter $b^{2}=\ell_{t} \ell_{r}$ is equal to $\left(\ell_{t} \ell_{r}\right)_{\max }$. Note that the maximum Cassini oval depends, through the bistatic constant $\Gamma$, on system parameters involving the $\mathrm{Tx}$ and the $\mathrm{Rx}$, the target, and the system noise; it also depends on the required performance (in terms of missed detection and false alarm) through the quantity $S N R_{\min }$. Any point belonging to the interior of the maximum Cassini oval is said to be covered by the bistatic pair, as for a target located in that point the required performance can be achieved. A point is said to be covered by an RSN whenever it is covered by a number of bistatic pairs that is sufficient to localize the target position. ${ }^{5}$

In a UWB bistatic pair, the estimate performed by the Rx of the time-of-arrival (ToA) of both the pulse received along the direct path and the pulse reflected by the target ${ }^{6}$ yields an estimate of the sum distance $\ell_{t}+\ell_{r}$. Based on it, the Rx is able to locate the target on an ellipsoid whose foci are in the Tx and $\mathrm{Rx}$ positions (hence, the distance between the foci equals the baseline $\ell$ ) and whose major axis equals the estimate of $\ell_{t}+\ell_{r}$. The intersection of the ellipsoid with any plane containing both the Tx and the Rx is an ellipse with the same foci and the same major axis, representing an iso-range (equivalently, an iso-ToA) curve for the Tx-Rx pair. Examples of iso-ToA curves are shown in Fig. 4 in which, similarly to Fig. 3, both

\footnotetext{
${ }^{5}$ In a two-dimensional environment the number of such bistatic pairs is typically equal to three, although the blind zone problem (see Sec. IV-G) may impose some redundancy.

${ }^{6}$ Examples of such procedures are described in Sec. IV-C and Sec. IV-E.
} 


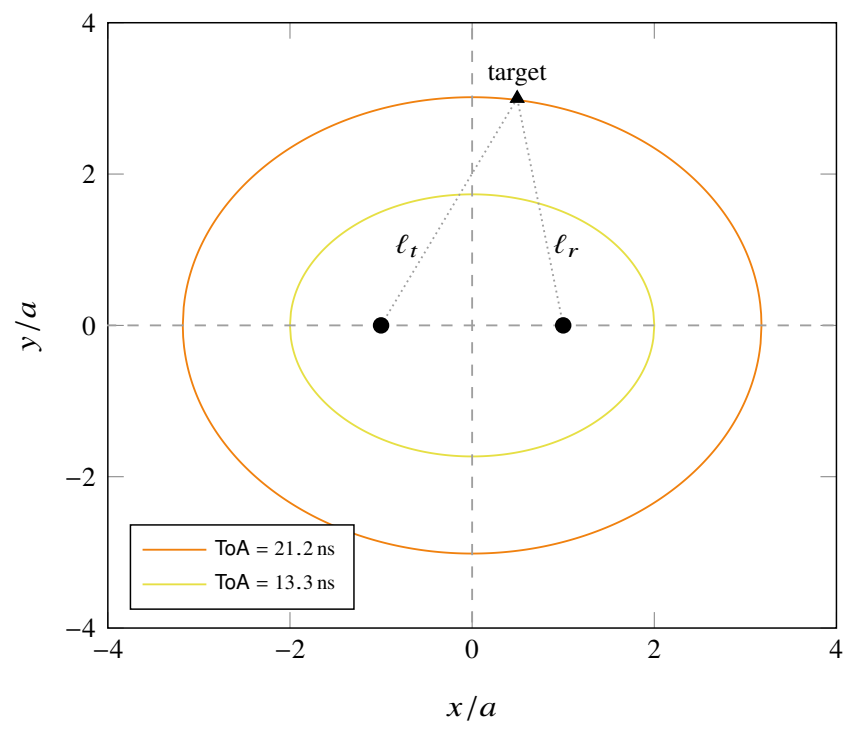

Fig. 4. Examples of iso-ToA ellipses for a bistatic pair. Both the $x$ - and the $y$-axis are normalized with respect to $a=\ell / 2$. The Tx and the $\mathrm{Rx}$ are in positions $(-\ell / 2,0)$ and $(\ell / 2,0)$. Inner curve: $\left(\ell_{t}+\ell_{r}\right) /(\ell / 2)=4$.0. Outer curve: $\left(\ell_{t}+\ell_{r}\right) /(\ell / 2)=6.4$. The ToA values in the legend are defined as $\left(\ell_{t}+\ell_{r}\right) / c$ and are relevant to the case $\ell / 2=1$.

axes are normalized with respect to $a=\ell / 2$. Importantly, over any plane containing the Tx and Rx positions iso-SNR curves (Cassini ovals) and iso-ToA curves (ellipses) do not coincide: the SNR changes when the target moves along any iso-range curve and, similarly, the target ToA changes when the target moves along any iso-SNR curve.

To see how the SNR changes along an iso-ToA curve, let us denote by $\beta$ the Tx-target-Rx angle (see Fig. 3). This angle, usually dubbed the bistatic angle, ranges between zero (when the target is located on the extended baseline) and $\beta_{\max }=2 \arcsin \left(\ell /\left(\ell_{t}+\ell_{r}\right)\right)$ along any iso-ToA ellipse. We have $\ell^{2}=\ell_{t}^{2}+\ell_{r}^{2}-2 \ell_{t} \ell_{r} \cos \beta$ from which, after simple algebraic manipulation we obtain

$$
\ell_{t} \ell_{r}=\frac{\left(\ell_{t}+\ell_{r}\right)^{2}-\ell^{2}}{2(1+\cos \beta)}
$$

and therefore, from (2),

$$
\operatorname{SNR}=\frac{4 \Gamma(1+\cos \beta)^{2}}{\left(\left(\ell_{t}+\ell_{r}\right)^{2}-\ell^{2}\right)^{2}} .
$$

Equation (7) expresses the SNR as a function of the bistatic angle $\beta$, of the bistatic radar constant $\Gamma$, and of the bistatic range $\ell_{t}+\ell_{r}$. When a target moves along any iso-ToA curve (for a constant value of $\Gamma$ ), the SNR is a decreasing function of $\beta$. Therefore, the SNR assumes its maximum value, equal to $16 \Gamma /\left(\left(\ell_{t}^{2}+\ell_{r}\right)^{2}-\ell^{2}\right)^{2}$, when the target is located on the extended baseline $(\beta=0)$; it assumes its minimum value, equal to $16 \Gamma /\left(\ell_{t}+\ell_{r}\right)^{4}$, when the target is equidistant from the two radio nodes $\left(\beta=\beta_{\max }\right)$.

Taking the dual approach, we can look at the bistatic range variation along an iso-SNR curve. From (7) we have

$$
\ell_{t}+\ell_{r}=\sqrt{\ell^{2}+2(1+\cos \beta) \sqrt{\Gamma / \mathrm{SNR}}}
$$

where now the bistatic angle $\beta$ may take all values in $[0, \pi]$ when $\sqrt{\ell_{t} \ell_{r}} \leq \ell / 2$ (two loops or lemniscate), and all values between 0 and $\beta_{\max }=2 \arcsin \left((\ell / 2)(\Gamma / \mathrm{SNR})^{1 / 4}\right)$ when $\sqrt{\ell_{t} \ell_{r}}>\ell / 2$ (single oval). From (8) we may conclude that for a constant SNR and constant $\Gamma$, the parameter $\ell_{t}+\ell_{r}$ takes its maximum value $\left(\ell^{2}+4 \sqrt{\Gamma / \mathrm{SNR}}\right)^{1 / 2}$ when the target is located on the extended baseline $(\beta=0)$. For $\sqrt{\ell_{t} \ell_{r}}>\ell / 2$ it takes its minimum value $\left(2(\Gamma / \mathrm{SNR})^{1 / 4}\right)$ when the target is equidistant from the two radio nodes $\left(\beta=\beta_{\max }\right)$. For $\sqrt{\ell_{t} \ell_{r}} \leq \ell / 2$, it takes its minimum value when the target is on the baseline $(\beta=\pi)$.

All of the above considerations have been carried out for free-space propagation and presence of AWGN only. Although in real propagation environments (especially indoor ones) the channel model deviates radically from the simple AWGN one, the above ideas turn out to remain essentially valid, as far as the path loss channel component is concerned. This fact is also supported by the reference channel model for UWB indoor (residential, office, industrial environments) and outdoor (suburban environment) propagation [123], featuring a frequency-dependent path loss model in the form

$$
\mathrm{PL}(f, d)=\mathrm{PL}_{0} \times\left(f / f_{c}\right)^{2(\kappa+1)}\left(d / d_{0}\right)^{\rho}
$$

where $f_{c}$ is the center-band frequency, $\mathrm{PL}_{0}$ is a constant term depending on the environment, the coefficient $\kappa$ models the frequency dependence of the channel, the coefficient $\rho$ is the path loss exponent, and $d_{0}=1 \mathrm{~m}$ is the reference distance between the Tx and the Rx. Averaging out the fading channel components in the received PSD, and therefore exploiting the above path loss model to modify (1), it is still possible to obtain an expression of the SNR in the form (2), upon appropriately modifying the definition of bistatic radar constant $\Gamma$, where the SNR decays with the $\ell_{t} \ell_{r}$ product as $\left(\ell_{t} \ell_{r}\right)^{-\rho}$.

\section{In-SENSOR PROCESSING}

This section describes the main processing steps carried out within a single sensor radar, focusing on the transmission between two sensors. Such steps may be summarized as pre-filtering of the received signal $y(t)$, coherent processing, envelope extraction, ToA estimation, clutter removal, ghost mitigation, CFAR detection, and one-dimensional clustering.

\section{A. Received signal}

With reference to Fig. 1, the received signal $y(t)$ is first processed by a band-pass filter, having the same center frequency and bandwidth as $y(t)$, to remove out-of-band noise spectral components. After filtering, the waveform associated with a frame may be written as ${ }^{7}$

$$
\widetilde{r}(t)=\sum_{n=0}^{N_{\mathrm{s}}-1} \sum_{l=0}^{L_{\mathrm{p}}-1} \gamma_{l} p_{l}\left(t-n T_{\mathrm{IP}}-\tau_{l}\right)+\widetilde{w}(t)
$$

\footnotetext{
${ }^{7}$ Since we consider UWB signals, equivalent baseband notation is not adopted and all signals are real.
} 
where $L_{\mathrm{p}}$ is the number of received multipath components, each with gain $\gamma_{l}$ and delay $\tau_{l}, p_{l}(t)$ is the $l$ th received pulse, ${ }^{8}$ and $\widetilde{w}(t)$ is the filtered AWGN. Note that the coefficients $\gamma_{l}$ are assumed to be constant over a frame, a realistic assumption in typical object tracking scenarios.

Next, let us denote by $r(t)$ the coherent average of the $N_{\mathrm{s}}$ pulses received within a frame. Such an averaging operation, also known as pulse integration, yields a significant improvement of the SNR at the receiver. This turns out to be very beneficial for the whole detection and localization process as object echoes may be very weak. After pulse integration the received signal relevant to a single frame, also referred to as a scan, may be written from (10) as

$$
r(t)=\sum_{l=0}^{L_{\mathrm{p}}-1} \gamma_{l} p_{l}\left(t-\tau_{l}\right)+w(t)
$$

Note that $w(t)$ has a power reduced by a factor $N_{\mathrm{s}}$ with respect to $\widetilde{w}(t)$ in (10), resulting in an SNR increase by the same factor. Note also that the scan waveform has a duration equal to $T_{\mathrm{IP}}$ but the collection of the $N_{\mathrm{s}}$ pulses to form a scan requires an entire frame of duration $T_{\mathrm{f}}$.

The received scan waveform (11) is sampled at frequency $f_{\mathrm{s}}=1 / T_{\mathrm{s}}$, and its samples are collected into a vector $\mathbf{r}=$ $\left(r_{1}, \ldots, r_{N}\right)$ of length $N=T_{\mathrm{f}} / T_{\mathrm{s}}$, where

$r_{k}=r\left(k T_{\mathrm{s}}\right)=\sum_{l=0}^{L_{\mathrm{p}}-1} \gamma_{l} p_{l}\left(k T_{\mathrm{s}}-\tau_{l}\right)+w\left(k T_{\mathrm{s}}\right) \quad k=1, \ldots, N$.

To better illustrate the in-sensor processing steps depicted in Fig. 1, we show in Fig. 5 an example of the signal captured, after each processing block, in a real indoor scenario through the test-bed described in Sec. VI. The sampling frequency is $f_{\mathrm{s}}=16.4 \mathrm{GHz}$ which corresponds to a sampling time $T_{\mathrm{s}}=61 \mathrm{ps}$. The number of pulses per frame is $N_{\mathrm{s}}=20480$ and the scan length is $N=1920$ samples. Note that the time required to collect samples for a scan is approximately 9 $N_{\mathrm{s}} N T_{\mathrm{s}}=2.4 \mathrm{~ms}$. Such a duration is small enough to ensure that the environment (including the target) is quasi-static and, therefore, that coherent integration is effective.

Fig. 5a shows the sampled scan waveform (12), $\mathbf{r}_{\mathrm{er}}$, when the target is not present, namely, in empty-room (ER) conditions. The scan presents a very high peak at ToA $\tau_{0}=20 \mathrm{~ns}$ due to the direct path (DP), as well as additional multipath components induced by reflections in the propagation environment. In presence of a target in the monitored area, the sampled scan waveform $\mathbf{r}$ may look like the red one in Fig. 5 b. As the figure emphasizes, the target produces an extra path, which is rather weak in the example waveform. This makes the subsequent steps crucial for reliable detection and precise localization of the object. The target echo ToA is $\tau=32.6 \mathrm{~ns}$.

\section{B. Non-coherent demodulation}

By processing vector $\mathbf{r}$, whose $k$ th element is given by (12), it is possible to extract the ToA $\tau_{0}$ of the DP. Since the

\footnotetext{
${ }^{8} p_{l}(t)$ may account for distortions due to antennas, reflections, frequencydependent RCS of the target, and filtering in the receiver front-end.

${ }^{9}$ The actual scan duration is slightly higher as an inter-frame period is present.
}

transmission is IR-based with a very large bandwidth, as the echo pulse is very short, ToA information may be extracted even relying on non-coherent demodulation techniques, i.e., the carrier phase needs neither to be estimated nor tracked [124]. This feature is very beneficial, as the pulse $p_{l}(t)$ is usually unknown at the receiver because of unavoidable distortions introduced by the channel and the antennas, due to its very large bandwidth. A simple envelope detector may thus be applied both to the ER scan $\mathbf{r}_{\mathrm{er}}$ and to the current scan $\mathbf{r}$ to extract their envelopes $\boldsymbol{\epsilon}_{\mathrm{er}}$ and $\boldsymbol{\epsilon} .{ }^{10}$ Envelope extraction may be easily implemented by a squaring block followed by a low-pass filter. The result is exemplified in Fig. 5c, where the target echo is again detailed.

\section{Direct path ToA estimation}

To perform clutter removal (see next subsection), after envelope extraction we need to provide an estimate $\hat{\tau}_{0}$ of the DP ToA, for both $\epsilon_{\mathrm{er}}$ and $\epsilon$. In fact, once the two DP ToA estimates are available, the two vectors can be aligned by shifting one of them by the appropriate amount $\left\lfloor\left|\hat{\tau}_{0}-\hat{\tau}_{0, \text { er }}\right| / T_{\mathrm{s}}\right\rfloor=\left\lfloor\left|\Delta \hat{\tau}_{0}\right| / T_{\mathrm{s}}\right\rfloor$.

There are numerous techniques for ToA estimation in UWBIR systems; a detailed review may be found in [47]. A simple technique to detect the first arriving path is threshold crossing, where the signal envelope is compared with a threshold and the first crossing gives the ToA [125], [126]. The threshold crossing detector performance is heavily influenced by the threshold value, which needs to be carefully designed according to the operating conditions. A simple criterion to determine a sub-optimal threshold, based on the evaluation of the probability of early detection and knowledge of noise power, may be found in [125]. Several other sub-optimum approaches have been proposed to reduce early detections threshold-based estimation such as jump back and search forward criterion (exploiting the detection of the strongest sample and a forward search procedure over a predefined time window), and serial backward search criterion (based on the detection of the strongest sample and a search-back procedure over a predefined time window) [126], [127]. A comprehensive framework for the analysis and design of threshold-based ToA estimators was developed in [128]. More robust techniques, which do not require knowledge of the signal statistics and noise power, are investigated in [49], [129].

\section{Clutter removal and ghost mitigation}

After alignment of the ER and the current scan, clutter removal is performed as ${ }^{11}$

$$
\mathbf{d}=\boldsymbol{\epsilon}-\boldsymbol{\epsilon}_{\mathrm{er}} .
$$

\footnotetext{
${ }^{10}$ As opposed to $\epsilon$, which is computed in each frame, ER envelope $\epsilon_{\mathrm{er}}$ is extracted only any time a new ER scan is acquired.

${ }^{11}$ In some cases, the alignment based on the DP ToA estimation of the two scans is not sufficiently accurate to perform an effective clutter removal. Since the two scans are very similar (see, e.g., Fig. 5c) cross-correlation peak searching is an effective approach to estimate the relative temporal shift used for a finer alignment. Such approach can be further improved by performing interpolation of the two scans at a higher sampling rate, before alignment, and then decimation after the scans subtraction, (13), to return to the initial sampling rate.
} 


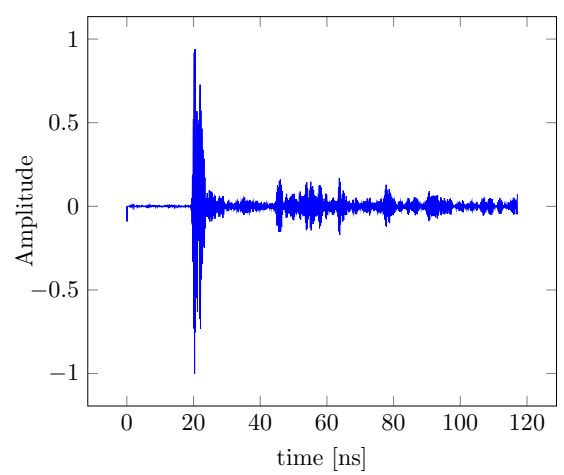

(a) Received vector $\mathbf{r}_{\mathrm{er}}$ relative to the ER.

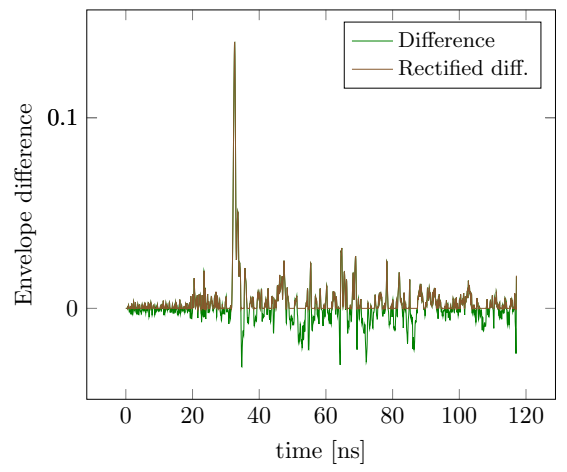

(d) Envelope difference after clutter removal and ghost rejection.

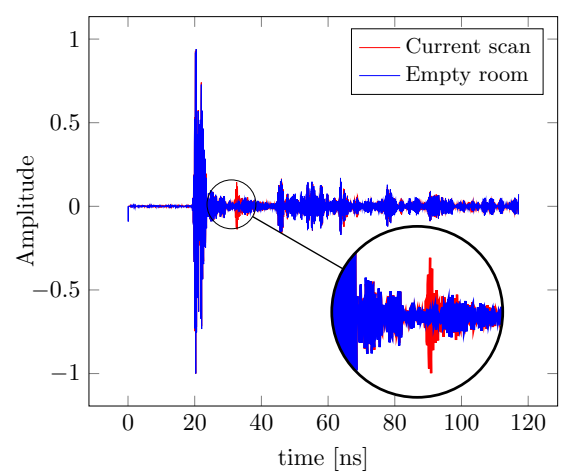

(b) ER and current scan waveforms.

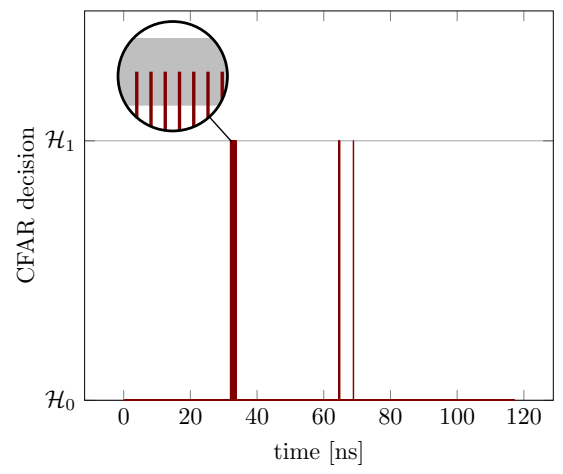

(e) Decisions after CFAR detection.

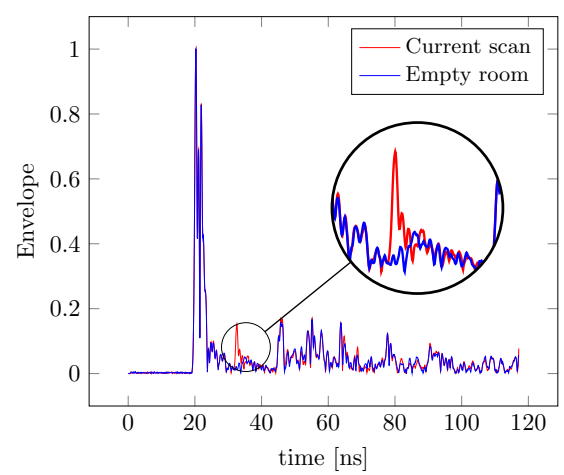

(c) Envelopes of the ER and the current scan.

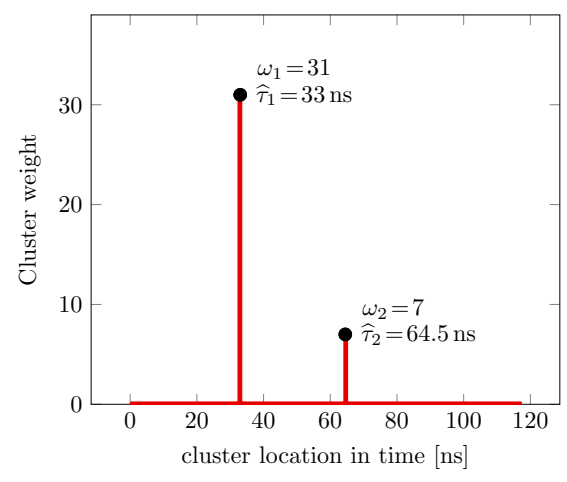

(f) Measurement vector after one-dimensional clustering.

Fig. 5. In-sensor processing steps at $\mathrm{Rx}_{4}$ for the $32 \mathrm{nd}$ scan in the reference scenario depicted in Fig. 7. The received waveform is processed following the steps from (b) to (f). The measurement vector at the output of the sensor will be processed by the fusion center as illustrated in Sec. V.

Vector $\mathbf{d}$ is expected to contain the target echo immersed in a clutter residue that was not removed due to imperfect DP ToA estimation and waveform alignment, and because clutter is non-static (e.g., due to moving foliage in outdoor or other undesired moving objects within the area). Apart from residual clutter, the obtained signal samples may also be impaired by additional echoes named ghosts, typically appearing when a target obscures a clutter echo which is present in the ER scan. Ghost echoes manifest as negative peaks in the difference vector (13). To mitigate ghost effects, a half-wave rectifier is applied to $\mathbf{d}$ (see an example in Fig. 5d). With some abuse of notation, hereafter the rectified vector is still denoted by $\mathbf{d}$.

In the presence of strong moving clutter and a moving target, several clutter removal techniques have been developed, often under the name moving target indicator (MTI), ranging from a simple frame-to-frame subtraction to more sophisticated approaches, some of which exploit features of the human motion [92], [130]-[134].

\section{E. CFAR detection}

Once the signal samples have been processed by the clutter removal and ghost mitigation algorithms, the rectified vector $\mathbf{d}$ is further processed to make a sample-wise decision between the null hypothesis, $\mathcal{H}_{0}$, and the alternative hypothesis, $\mathcal{H}_{1}$, thus generating a binary vector $\hat{\mathbf{b}}=\left(\hat{b}_{1}, \hat{b}_{2}, \ldots, \hat{b}_{N}\right)$ to be processed by the subsequent steps.
A naïve sample-wise detector consists of comparing each sample with a fixed threshold. If the sample under test (SUT), $d_{k}$, is above the threshold, the corresponding decision vector sample $\hat{b}_{k}$ will be set to 1 , otherwise $\hat{b}_{k}=0$. The choice of the threshold is crucial and may depend on several aspects, such as noise and residual clutter powers as well as their statistics, the number and location of the targets, etc. It is worth noting that increasing the threshold causes false alarm rate to reduce but at the same time misdetections to raise, and vice versa.

CFAR detectors feature an adaptive threshold aimed at maximizing the detection probability while keeping the false alarm rate approximately constant [135]. The most well-known approach is cell averaging CFAR (CA-CFAR) detection [26], [119], [136], [137]. Here the sample-specific threshold is calculated by estimating the level of residual clutter and noise around the SUT. The estimation is performed by considering two blocks of reference samples (of length $N_{\text {ref }}$ samples each) around the SUT (one on the right and the other on the left of it) and by calculating their average power ignoring $N_{\text {guard }}$ guard samples immediately adjacent to $d_{k}$. Guard samples are discarded to avoid over-estimating the clutter and noise power due to the target itself. In fact, the target echo usually involves several samples as exemplified in Fig. 5c. Then this average power is compared with the squared SUT multiplied by the 
threshold $\alpha$ as

$$
d_{k}^{2} \underset{\mathcal{H}_{0}}{\stackrel{\mathcal{H}_{1}}{\gtrless}} \alpha \frac{\sum_{i \in \mathcal{S}_{k}} d_{i}^{2}}{2 N_{\text {ref }}} \quad k=1, \ldots, N
$$

where $\mathcal{S}_{k}=\left\{k-N_{\text {guard }}-N_{\text {ref }}, \ldots, k-N_{\text {guard }}-1, k+N_{\text {guard }}+\right.$ $\left.1, \ldots, k+N_{\text {guard }}+N_{\text {ref }}\right\} \cap\{1,2, \ldots, N\}$ is the set of reference samples.

A drawback of CA-CFAR is target masking, a problem arising when another target falls into the reference set $\mathcal{S}_{k}$, thus increasing considerably the reference power and leading to a wrong decision for $\mathcal{H}_{0}$. To overcome such a limitation, several variants of the CA-CFAR detector have been introduced, depending on the specific type of waveform and on the scenario. A simple variation of CA-CFAR is global CFAR (G-CFAR) where the reference samples are extended to all scan samples except for the guard ones. Formally, the detector still has the structure (14) in which $\mathcal{S}_{k}=$ $\left\{1, \ldots, k-N_{\text {guard }}-1, k+N_{\text {guard }}+1, \ldots, N\right\}$. This way the SUT is compared with an estimated power which depends on the whole scan (and not on local reference samples), thus averaging out the echoes from the other targets over a larger interval. This approach turns out to be effective only when the number of targets inside the monitored area is small and the scan period is long enough. As a drawback, moreover, the information associated with neighbor samples, which could be useful for a better decision based on local residual clutter, is now overlooked. This last aspect is quite important, as in an indoor scenario clutter is non-homogeneous [138]-[140]. In these contexts, a more sophisticated variant of CA-CFAR, named ordered statistics (OS-CFAR), performs ordering of the reference samples $\mathcal{S}_{k}$ according to their magnitude and by selecting a certain predefined value from the ordered sequence [135]. Thanks to ranking, OS-CFAR is proven to be more robust than CA-CFAR and G-CFAR in the presence of outliers, has happens in non-homogeneous clutter.

In the context of UWB indoor radar tracking, a recent proposal called double threshold and buffer CFAR (DTB-CFAR) allows overcoming the above limitations by means of an adaptive threshold setting strategy [65]. For each sample $d_{k}$ of the rectified vector $\mathbf{d}$, the detector takes a binary decision

$$
d_{k}^{2} \underset{\mathcal{H}_{0}}{\stackrel{\mathcal{H}_{1}}{\gtrless}} \alpha_{1} \mathcal{L}_{\mathrm{p}}+\alpha_{\mathrm{g}} \mathcal{G}_{\mathrm{p}} \quad k=1, \ldots, N
$$

where $\mathcal{L}_{\mathrm{p}}$ and $\mathcal{G}_{\mathrm{p}}$ are defined as the local and the global power respectively, whereas $\alpha_{1}$ and $\alpha_{\mathrm{g}}$ are weighting factors. The reference powers $\mathcal{L}_{\mathrm{p}}$ and $\mathcal{G}_{\mathrm{p}}$, and thus the threshold $\eta=\alpha_{1} \mathcal{L}_{\mathrm{p}}+\alpha_{\mathrm{g}} \mathcal{G}_{\mathrm{p}}$, are calculated through Algorithm 1. In particular, $\mathcal{G}_{\mathrm{p}}=(1 / N) \sum_{i=1}^{N} d_{i}^{2}$ is the average power of the rectified vector $\mathbf{d}$, whereas $\mathcal{L}_{\mathrm{p}}$ is the average power of a first in first out buffer, $\mathbf{s}=\left(s_{1}, \ldots, s_{N_{\mathrm{b}}}\right)$, of length $N_{\mathrm{b}}$, filled with the already processed $\mathbf{d}$ samples that were found to be below threshold. This way we reduce the misdetection rate in case of multiple-target scenarios as we keep track of the decisions previously taken during the scan. Keeping $N_{\mathrm{b}}$ short, it is possible to get a local estimate of the noise and residual clutter power level for the near-SUT samples. However, since target echo has a length of several samples, the overall threshold (due to $\mathcal{L}_{\mathrm{p}}$ ) increases very quickly, causing misdetections. To avoid this phenomenon, if the increment of the SUT with respect to the previous sample is larger than a predefined value, $\Delta \eta$, for at least $q_{\max }$ consecutive samples, then $\mathcal{L}_{\mathrm{p}}$ is not updated. Thus, since residual clutter and target presence are characterized by similar echoes with the only difference that the latter ones are generally significantly larger than the former ones, $\eta$ is expected to increase quickly enough to avoid false alarms but also be frozen soon enough to properly detect the target. Later, the value of $\mathcal{L}_{\mathrm{p}}$ is unfrozen when a waveform decrease occurs.

To further decrease misdetections, because of the asymmetric target echo, DTB-CFAR is applied to the scan twice in opposite directions. Then, the two outputs are merged through an element-wise OR operator to form the final vector. It has been experimentally verified that DTB-CFAR provides better performance than conventional approaches (e.g., CA-CFAR) in case of multiple human targets in densely cluttered environments. Hereafter, we denote by $\hat{b}$ the binary vector generated by DTB-CFAR from processing of $\mathbf{d}$ (see Fig. 1). An example is depicted in Fig. 5e when $\alpha_{1}=5, \alpha_{\mathrm{g}}=8, N_{\mathrm{b}}=25$, and $q_{\max }=4$. In the example, $\hat{\mathbf{b}}$ exhibit three bursts of ' 1 's corresponding to three possible targets. Such vector will be further processed within the sensor by one-dimensional clustering. ${ }^{12}$

\section{F. One-dimensional clustering}

The generic vector $\hat{\mathbf{b}}$ returned by the detector is typically characterized by bursts of ' 1 's, each due to a specific target or (to a lesser extent) to residual clutter. To further filter out false alarms due to residual clutter and limit the computational complexity of network processing, a clustering algorithm is applied to the vector $\mathbf{b}$, resulting in a new binary vector denoted by $\mathbf{b}$. We call this clustering step one-dimensional clustering (see Fig. 1). Since no a priori knowledge of the number of clusters is available, hierarchical clustering is an effective approach [141], [142].

In the beginning, each ' 1 ' forms a singleton cluster. Then, iteratively as long as the minimum distance between two clusters is less than a preset distance threshold, $d_{\text {thr }}$, the two clusters at minimum distance are merged. ${ }^{13}$ At the end, each survived cluster is replaced by a single ' 1 ' in the median position. Furthermore, a weight $\omega$ equal to the number of clusterized ' 1 's is associated with every final cluster. As the clusters associated with targets typically exhibit higher weights than those related to clutter or noise, it is useful to set also a weight-based threshold, $\omega_{\min }$, so that the lightest survived clusters (having a weight less than $\omega_{\min }$ ), are discarded and the corresponding elements of $\mathbf{b}$ are set to ' 0 '.

\footnotetext{
${ }^{12}$ It is worthwhile remarking that the vector $\hat{\mathbf{b}}$ computed by each sensor node contains detection information for all targets in the area. As the quality of the channel between the Tx, a specific target, and a specific Rx depends on the target position (e.g., this is true for the SNR given by (2)), different sensor nodes are likely to deliver detection information with a different quality per each target. This consideration applies to any in-sensor detector.

${ }^{13} \mathrm{We}$ define the distance between two clusters as the distance between their centroids (i.e., the median positions among the '1's which compose those clusters at the current iteration).
} 


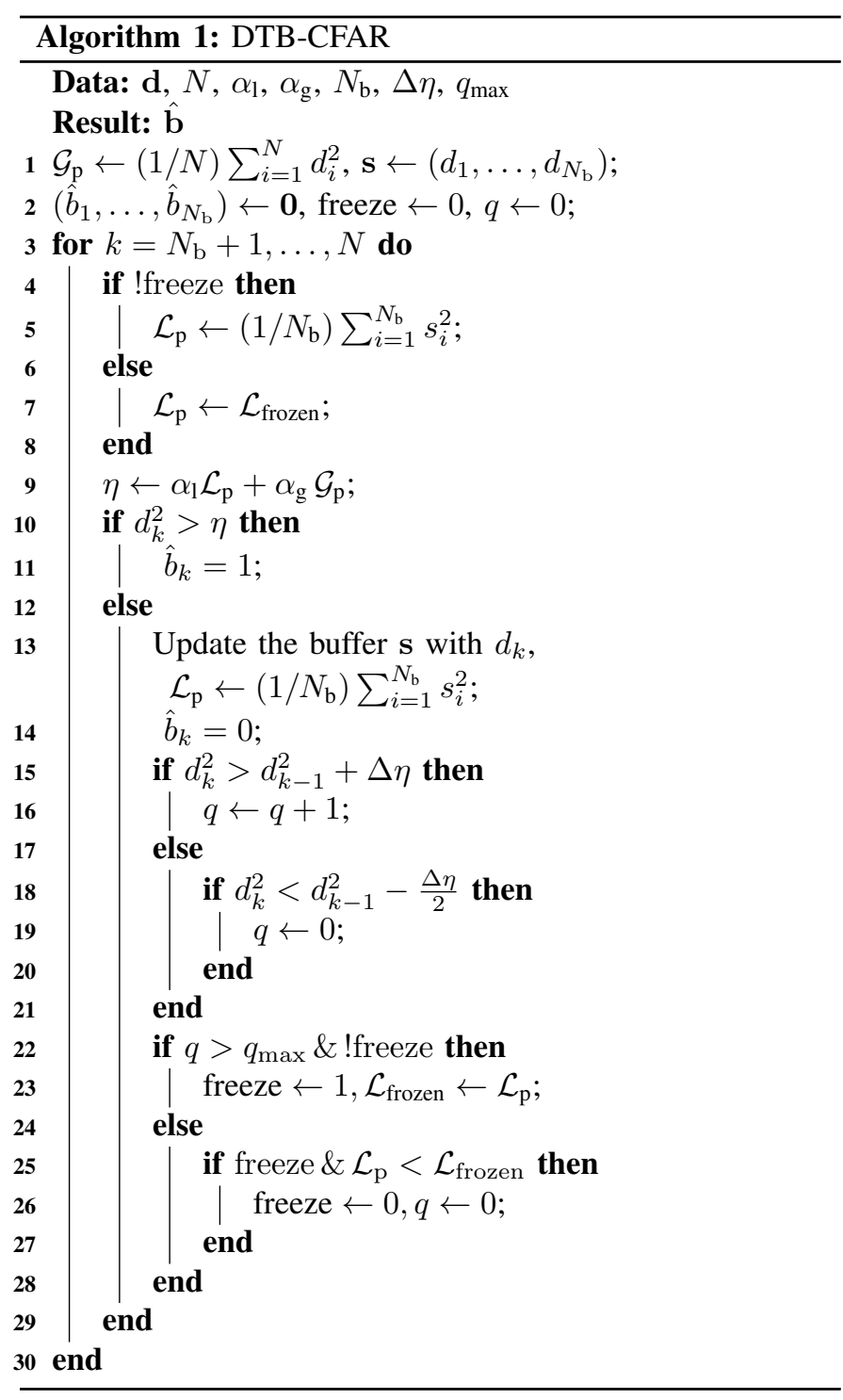

The output of one-dimensional clustering is exemplified in Fig. 5f, where the weight threshold is set to $\omega_{\min }=4$ and the minimum distance threshold is $d_{\mathrm{thr}}=65$ samples, corresponding to $65 T_{\mathrm{s}}=3.97 \mathrm{~ns}$. Note that the estimated ToA of the target echo is $\hat{\tau}=33 \mathrm{~ns}$, which compared to the actual one, $\tau=32.6 \mathrm{~ns}$, confirms the good accuracy. However, there is a second ' 1 ' in vector $\mathbf{b}$ which corresponds to a false alarm. Such an undesired measurement will be further processed and hopefully filtered out by network processing.

We report in Fig. 6 the temporal evolution of the scan waveforms obtained after clutter removal, DTB-CFAR detection, and one-dimensional clustering by one of the sensor nodes $\left(\mathrm{Rx}_{4}\right.$ in Fig. 7), in the framework of the case study that will be described in Sec. VI. Specifically, Fig. 6a shows the evolution of the actual (single) target ToA, while Fig. 6b, Fig. 6c, and Fig. 6d show vertically stacked vectors $d, \hat{b}$, and b, respectively. Looking at Fig. 6d, we can see how the target is detected in almost all frames (filling the gaps is a problem deferred to tracking); false alarms in some of the frames are also visible.

\section{G. The blind zone problem}

An issue deserving attention in sensor radar systems based on UWB is represented by the existence of a blind zone for each bistatic Tx-Rx pair, i.e., a region in the proximity of the direct path between the Tx and the Rx where target detection becomes problematic. Blind zones are due to concurrent phenomena, as explained next.

A non-point-wise target (e.g., a human being) becomes virtually undetectable by a bistatic pair when it is located on the path between the Tx and the Rx node. Due to target obstruction of the LOS between the two nodes, neither a detectable direct signal nor a detectable target-scattered one are received; whenever a detectable target echo is still received, it is assumed to represent the direct signal.

A target is generally undetectable also when it is sufficiently close to the LOS between the two bistatic nodes, although the LOS is not shadowed by it. This is a consequence of synchronization issues. Synchronization based on estimation of the ToA of the direct-path pulse is the first operation performed by the Rx node. This operation is essential both to align the $N_{s}$ pulse responses belonging to the same frame (to achieve pulse integration gain) and to align successive frames for clutter removal purposes, as addressed in Sec. IV-C. In practice, imperfect ToA estimation turns into imperfect frame alignment and, consequently, presence of a clutter residue after clutter removal. Since the signal received along the direct path is usually much stronger than the target echo and the other clutter components, a direct-path pulse residue after clutter removal is normally comparable to the echo of a target close to the direct path between the $\mathrm{Tx}$ and the $\mathrm{Rx}$, if not even stronger. ${ }^{14}$ Due to residual clutter issues, received signal samples with small delay with respect to the estimated ToA are usually considered as unreliable and ignored for detection purposes.

\section{Network Processing}

In Sec. IV we described a non-coherent detection algorithm performed by each Rx node composing the sensor radar. This algorithm yields, for each such node, a sparse binary vector $\mathbf{b}$ in which each nonzero entry is associated with a detected moving target in the surveilled area or with a false alarm. The next step consists of network processing, in which the harddecision information individually generated by all $\mathrm{Rx}$ nodes are fused together in order to complete target detection and tracking. A set of measurements is first generated and then a target tracking block is fed with such measurements. This section focuses on a set of low-complexity network processing algorithms.

\section{A. Detection (measurement generation)}

For ease of exposition we restrict the analysis to a twodimensional scenario, the extension to a three-dimensional one being immediate. The detection step consists of generating a

\footnotetext{
${ }^{14}$ It is worth mentioning that a similar masking effect may experimentally be observed when the ToA of the target echo is close to the ToA of the echo of any object characterized by a considerably larger RCS.
} 


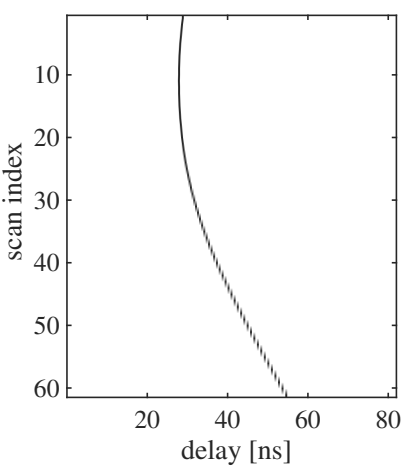

(a)

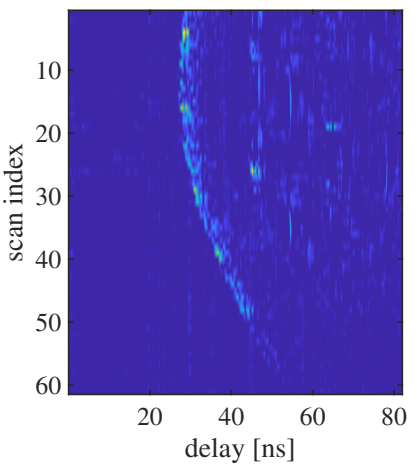

(b)

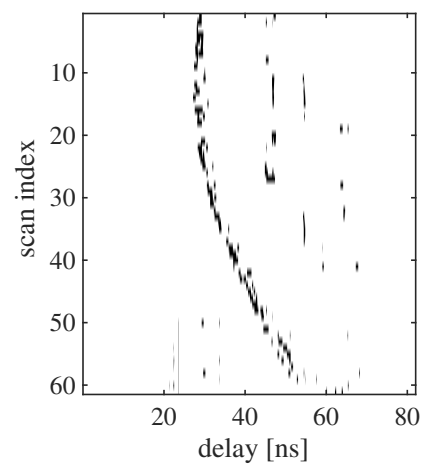

(c)

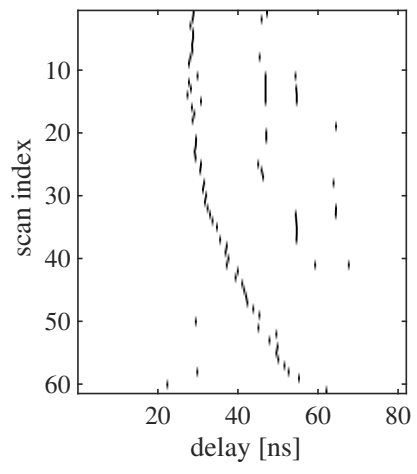

(d)

Fig. 6. Processing steps at $\mathrm{Rx}_{4}$ for the $N_{\text {scan }}=61$ scans collected along the target trajectory depicted in Fig. 7: (a) Actual target ToA; (b) Vectors $\mathbf{d}$ after clutter removal and rectification, where the lighter the color the larger the value of the corresponding sample; (c) Binary vectors $\hat{\mathbf{b}}$ after DTB-CFAR detection; (d) Binary vectors $\mathbf{b}$ after one-dimensional clustering.

set of $(x, y)$ pairs, each of which represents a measurement for the current scan period. Detection starts from the $N_{\mathrm{r}}$ binary vectors $\mathbf{b}_{i}, i \in\left\{1,2, \ldots, N_{\mathrm{r}}\right\}$, each one returned by a sensor node in the network, and from the knowledge of the absolute positions of all nodes ( $\mathrm{Tx}$ and $\mathrm{Rx}$ ) in the network. As every nonzero entry in each vector is associated with a ToA value, and therefore with an iso-ToA curve having the corresponding Tx and Rx nodes as foci, target detection may be brought back to an ellipse intersection problem.

Let $\left(x_{t}, y_{t}\right)$ and $\left(x_{i}, y_{i}\right)$ denote the absolute coordinates of the Tx node and of the $i$ th $\mathrm{Rx}$ node, $i \in\left\{1,2, \ldots, N_{\mathrm{r}}\right\}$, and let us consider the binary vector $\mathbf{b}_{i}$ returned by the $i$ th $\mathrm{Rx}$ node during the current scan. Moreover, let us index by $h$ the nonzero entries in $\mathbf{b}_{i}$, and denote by $\hat{\tau}_{i, h}$ the corresponding estimated ToA. Based on the $h$ th nonzero entry of $\mathbf{b}_{i}$, the fusion center locates a target on the ellipse whose foci are in the positions $\left(x_{t}, y_{t}\right)$ and $\left(x_{i}, y_{i}\right)$, and whose major axis is $\hat{q}_{i, h}=\ell_{i}+c \hat{\tau}_{i, h}$, where $\ell_{i}$ is the $i$ th baseline. The equation of the ellipse (iso-ToA curve) on which the fusion center locates the target based on the $h$ th nonzero entry of $\mathbf{b}_{i}$ is therefore

$\sqrt{\left(z_{x}-x_{i}\right)^{2}+\left(z_{y}-y_{i}\right)^{2}}+\sqrt{\left(z_{x}-x_{t}\right)^{2}+\left(z_{y}-y_{t}\right)^{2}}=\hat{q}_{i, h}$

where the generic point in the plane has been denoted by $\left(z_{x}, z_{y}\right)$. Squaring and reordering yields

$$
\begin{aligned}
z_{x}\left(x_{i}-x_{t}\right)+z_{y}\left(y_{i}-y_{t}\right)+p_{i, h} \\
=\hat{q}_{i, h} \sqrt{\left(z_{x}-x_{t}\right)^{2}+\left(z_{y}-y_{t}\right)^{2}}
\end{aligned}
$$

where

$$
p_{i, h}=\frac{1}{2}\left(\hat{q}_{i, h}^{2}-x_{i}^{2}-y_{i}^{2}+x_{t}^{2}+y_{t}^{2}\right) .
$$

We therefore obtain one equation in the form (17) for each Rx node $i \in\left\{1,2, \ldots, N_{\mathrm{r}}\right\}$ and for each nonzero entry in $\mathbf{b}_{i}$. A least square (LS) approach followed by a filtering step to limit false alarms and by a further clustering step is then employed in order to generate the measurements. The LS approach is described in the remainder of this subsection and the other two steps in Sec. V-B and Sec. V-C, respectively.
A LS solution may be found by selecting $\nu$ sensor nodes and one nonzero entry in each of the corresponding $\nu$ binary vectors, where $3 \leq \nu \leq N_{\mathrm{r}}$. To this aim, let us denote by $\mathcal{I} \subseteq\left\{1,2, \ldots, N_{\mathrm{r}}\right\}$ the set of indexes of the selected Rx nodes and by $h_{i}, i \in \mathcal{I}$, the index of the nonzero entry chosen in $\mathbf{b}_{i}$. (Recall that associated with $h_{i}$ we have an ellipse with foci in $\left(x_{t}, y_{t}\right)$ and $\left(x_{i}, y_{i}\right)$ and major axis $\left.\hat{q}_{i, h_{i}}.\right)$ Letting $k \in \mathcal{I}$, for $i=k$ equation (17) becomes

$$
\begin{aligned}
z_{x}\left(x_{k}-x_{t}\right)+z_{y}\left(y_{k}-y_{t}\right)+p_{k, h_{k}} \\
=\hat{q}_{k, h_{k}} \sqrt{\left(z_{x}-x_{t}\right)^{2}+\left(z_{y}-y_{t}\right)^{2}} .
\end{aligned}
$$

Next, for each $i \in \mathcal{I} \backslash\{k\}$ let us take the side-by-side difference between (17) multiplied by $\hat{q}_{k, h_{k}}$ and (19) multiplied by $\hat{q}_{i, h_{i}}$. After simple algebraic manipulation, this leads to the $N_{\mathrm{r}}-1$ equations

$$
a_{i, h_{i} ; k, h_{k}} z_{x}+b_{i, h_{i} ; k, h_{k}} z_{y}=g_{i, h_{i} ; k, h_{k}}
$$

where

$$
\begin{aligned}
a_{i, h_{i} ; k, h_{k}} & =\hat{q}_{i, h_{i}}\left(x_{k}-x_{t}\right)-\hat{q}_{k, h_{k}}\left(x_{i}-x_{t}\right) \\
b_{i, h_{i} ; k, h_{k}} & =\hat{q}_{i, h_{i}}\left(y_{k}-y_{t}\right)-\hat{q}_{k, h_{k}}\left(y_{i}-y_{t}\right) \\
g_{i, h_{i} ; k, h_{k}} & =\hat{q}_{k, h_{k}} p_{i, h_{i}}-\hat{q}_{i, h_{i}} p_{k, h_{k}} .
\end{aligned}
$$

The previous equations (20) may be recast in compact vector notation as

$$
\text { A } z=g
$$

where $\boldsymbol{z}=\left[z_{x} z_{y}\right]^{T}, \mathbf{A}$ is a $(t-1) \times 2$ matrix whose $i$ th row, $i \in \mathcal{I} \backslash\{k\}$, is given by $\left[a_{i, h_{i} ; k, h_{k}} b_{i, h_{i} ; k, h_{k}}\right]$, and $\boldsymbol{g}$ is a column vector with $\nu-1$ elements whose $i$ th component, $i \in \mathcal{I} \backslash\{k\}$, is $g_{i, h_{i} ; k, h_{k}}$.

Finally, using a LS approach, the target coordinates may be estimated as

$$
\begin{aligned}
\hat{\boldsymbol{z}} & =\arg \min _{\boldsymbol{z}}\|\mathbf{A} \boldsymbol{z}-\boldsymbol{g}\| \\
& =\left(\mathbf{A}^{T} \mathbf{A}\right)^{-1} \mathbf{A}^{T} \boldsymbol{g}
\end{aligned}
$$

where $\|\cdot\|$ is the Euclidean norm. A measurement $\hat{z}$ may be generated by applying (23) for each choice of the $3 \leq \nu \leq$ 
$N_{\mathrm{r}}$ nonzero elements, one per binary vector $\mathbf{b}$ created by a specific sensor node as described in Sec. IV. The approach here followed consists of choosing a value of $\nu$, considering all possible $\nu$-tuples of nonzero vector elements, one per $\mathrm{Rx}$ node, and generating one LS measurement per each $\nu$-tuple. The number of measurements generated with this approach is denoted by $N_{\mathrm{d}}$ in Fig. 1 .

The described measurement generation method inherently creates a relatively large number of false alarms. If no target is present in the area, false alarms are due to LS solutions combining nonzero vector elements caused by noise or clutter residues (i.e., by local false alarms at the $\nu$ Rx nodes). We will call these false alarms as type- 1 false alarms. When only one target is present, additional false alarms are due to LS solutions that combine nonzero vector elements associated with the target and nonzero vector elements being local false alarms. These false alarms will be referred to as type- 2 false alarms. Finally, when multiple targets are present, additional false alarms are generated by LS solutions that combine nonzero vector elements associated with different targets. False alarms of this type will be referred to as type- 3 false alarms.

The behavior of the detection algorithm (in terms of missed detections and number of generated false alarms) is sensitive to choice of the parameter $\nu$. To see this, we can look for example at the number of generated type-3 false alarms. Under the assumption that all $M \geq 2$ targets are detected by each of the $\nu$ $\mathrm{Rx}$ nodes and that each target corresponds to a unique nonzero element in each vector (perfect one-dimensional clustering), the number of type- 3 false alarms is readily given by

$$
N_{\mathrm{fa}, 3}=\frac{N_{\mathrm{r}} !}{\nu !\left(N_{\mathrm{r}}-\nu\right) !}\left(M^{\nu}-M\right) .
$$

For $N_{\mathrm{r}}=3, \nu$ is constrained to be equal to 3 yielding $N_{\mathrm{fa}, 3}=$ $M^{3}-M$. For $N_{\mathrm{r}}=4$, if $M \in\{2,3\}$ then a smaller number of type- 3 false alarms is attained by $\nu=4$, while it is attained by $\nu=3$ for any $M \geq 4$. For $N_{\mathrm{r}} \geq 5$, there exists $\bar{M}=\bar{M}\left(N_{\mathrm{r}}\right)$ such that: if $M \leq \bar{M}$ then when $\nu$ is increased from 3 to $N_{\mathrm{r}}$ the value of $N_{\mathrm{fa}, 3}$ initially increases, then it reaches a peak and finally decreases; if $M>\bar{M}$ then the value of $N_{\mathrm{fa}, 3}$ increases monotonically with $\nu \in\left\{3,4, \ldots, N_{\mathrm{r}}\right\}$.

\section{B. Decreasing the false alarm rate}

Due to the relatively large number of false alarms generated by the LS-based detector, a method to substantially reduce the false alarm rate at the input of the tracking filter becomes of fundamental importance. In this respect, a measurement selection is performed by introducing a notion of reliability for LS solutions. This is possible by exploiting both the weight $\omega$ associated with each nonzero entry in each of the $N_{\mathrm{r}}$ binary vectors $\mathbf{b}_{i}$ at the end of the in-sensor processing, and the residual error (RE), a measure of reliability inherently provided by the LS approach.

The RE associated with a LS solution (23) is

$$
\operatorname{RE}(\hat{\boldsymbol{z}})=\|\mathbf{A} \hat{\boldsymbol{z}}-\boldsymbol{g}\| .
$$

The idea is to keep a measurement if and only if its associated weight is above some threshold and its associated RE is below some other threshold, where both thresholds are invariant for all measurements. The weight of a measurement is defined as the sum of the weights of the $\nu$ nonzero vector entries yielding that measurement through (23), i.e., $\Omega=\sum_{i \in \mathcal{I}} \omega_{i}$. Setting a threshold $\Omega_{\text {th }}$ on the minimum acceptable measurement weight is effective in filtering out type- 1 false alarms and those type- 2 false alarms that are mostly contributed by local false alarms at Rx nodes. Type- 3 false alarms, however, are most often characterized by a weight above $\Omega_{\mathrm{th}}$ since they arise as combinations of local detections of different targets. Setting a threshold $R E_{\text {th }}$ on the maximum tolerable $\mathrm{RE}$ is effective in filtering out also type- 3 false alarms.

The number of surviving measurements after comparison of their weight and RE on the corresponding thresholds is denoted by $N_{\mathrm{m}} \leq N_{\mathrm{d}}$. For the sake of notational simplicity, surviving measurements will be simply denoted by $\boldsymbol{z}_{1}, \boldsymbol{z}_{2}, \ldots, \boldsymbol{z}_{N_{\mathrm{m}}}$ (see Fig. 1). Residual false alarms are possible even after the described selection procedure. In particular, while type- 1 false alarms are usually filtered out, the type2 and type- 3 ones being the result of combining some local detections of the same target might survive. Since these false alarms tend to generate measurements around the corresponding target position, a two-dimensional clustering operation is effective in reducing their rate. Two-dimensional clustering is also useful in presence of imperfect one-dimensional clustering.

\section{Measurement clustering}

The $N_{\mathrm{m}}$ measurements passing the false alarm reduction step tend to form clouds of points; this is particularly true when the number of sensors in the radar network is larger than three. Since the complexity of the processing to be performed in the subsequent tracking and data association steps is dependent on the number of measurements incoming from the detector, a further clustering operation (besides the one performed at node level) is applied to measurements. The approach is again hierarchical, iterative, and without an a priori specified number of clusters. It relies on the concept of distance between two clusters, for which several definitions are in principle possible.

Letting $\mathscr{Z}_{1}=\left\{\boldsymbol{z}_{1,1}, \boldsymbol{z}_{1,2}, \ldots, \boldsymbol{z}_{1, n}\right\}$ and $\mathscr{Z}_{2}=$ $\left\{\boldsymbol{z}_{2,1}, \boldsymbol{z}_{2,2}, \ldots, \boldsymbol{z}_{2, m}\right\}$ be two clusters of measurements with cardinality $n$ and $m$, respectively, a common notion of distance between $\mathscr{Z}_{1}$ and $\mathscr{Z}_{2}$, denoted by $d_{c}^{(c)}\left(\mathscr{Z}_{1}, \mathscr{Z}_{2}\right)$, is

$$
d_{c}^{(c)}\left(\mathscr{Z}_{1}, \mathscr{Z}_{2}\right)=\left\|\boldsymbol{c}\left(\mathscr{Z}_{1}\right)-\boldsymbol{c}\left(\mathscr{Z}_{2}\right)\right\|
$$

where $c(\mathscr{Z})$ is the centroid of cluster $\mathscr{Z}$. It is defined as

$$
\boldsymbol{c}(\mathscr{Z})=\frac{1}{|\mathscr{Z}|} \sum_{\boldsymbol{z} \in \mathscr{Z}} \boldsymbol{z}
$$

and in general does not coincide with any element of the cluster.

As mentioned, (26) is not the only possible definition of distance between clusters, as at least two other definitions are of practical interest. The first one measures the distance between two clusters $\mathscr{Z}_{1}$ and $\mathscr{Z}_{2}$ as the minimum Euclidean 


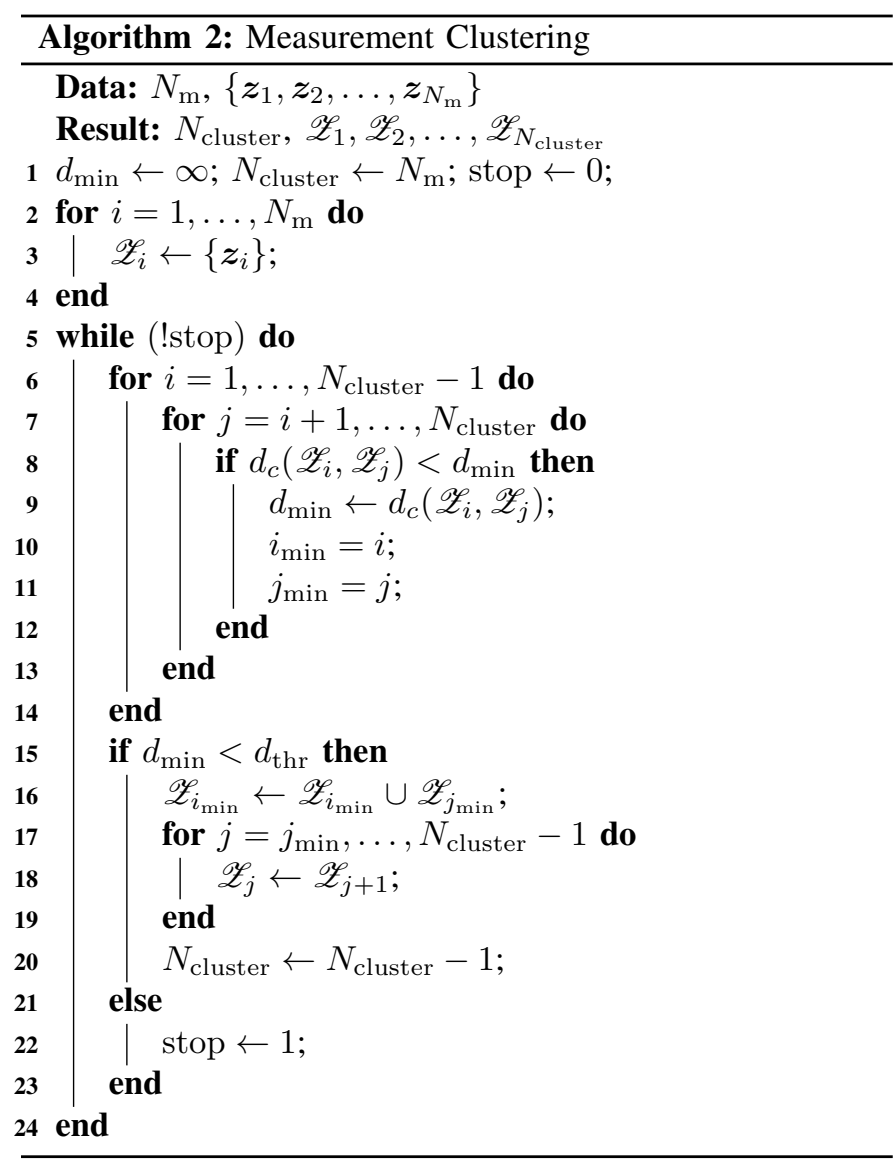

distance between one element $\boldsymbol{z}_{1} \in \mathscr{Z}_{1}$ and one element $\boldsymbol{z}_{2} \in \mathscr{Z}_{2}$, namely,

$$
d_{c}^{(m)}\left(\mathscr{Z}_{1}, \mathscr{Z}_{2}\right)=\min _{\left(\boldsymbol{z}_{1}, \boldsymbol{z}_{2}\right) \in \mathscr{Z}_{1} \times \mathscr{Z}_{2}}\left\|\boldsymbol{z}_{1}-\boldsymbol{z}_{2}\right\| .
$$

The second one is similar but relies on the maximum Euclidean distance between any two measurements of which one belongs to the first cluster and the other to the second:

$$
d_{c}^{(M)}\left(\mathscr{Z}_{1}, \mathscr{Z}_{2}\right)=\max _{\left(\boldsymbol{z}_{1}, \boldsymbol{z}_{2}\right) \in \mathscr{Z}_{1} \times \mathscr{Z}_{2}}\left\|\boldsymbol{z}_{1}-\boldsymbol{z}_{2}\right\| .
$$

The measurement clustering procedure is formalized in Algorithm 2. As also shown in Fig. 1, its input is represented by the set of all measurements generated by the detection block and surviving the false alarm rate reduction step, along with its cardinality $N_{\mathrm{m}}$; its output is a partition of this set along with the number of parts (i.e., clusters) $N_{\text {cluster }}$ In the beginning each measurements $\boldsymbol{z}_{i}$ forms a singleton cluster $\left\{\boldsymbol{z}_{i}\right\}$ and therefore $N_{\text {cluster }}=N_{\mathrm{m}}$. At each iteration, the minimum distance $d_{\min }$ between any two clusters is computed and compared with a threshold $d_{\mathrm{thr}}$; if $d_{\mathrm{min}}<d_{\mathrm{thr}}$ then the two corresponding clusters are merged to form a new cluster of larger cardinality and the number of clusters $N_{\text {cluster }}$ is decreased by one. The algorithm terminates when no pair of clusters is found whose distance is smaller than the threshold. The $N_{\text {cluster }}$ centroids of the finals clusters are forwarded to the multitarget tracking algorithm (Sec. V-D).

From a complexity viewpoint, during the first iteration of the algorithm, the one working on singleton clusters, we need to compute $\left(\begin{array}{c}N_{\mathrm{m}} \\ 2\end{array}\right)$ Euclidean distance values. Moreover, if the centroid-based definition of distance between clusters is adopted, during each subsequent iteration we need to calculate one centroid (for the new cluster formed during the previous iteration) through (27) and $N_{\text {cluster }}-1$ Euclidean distance values, where $N_{\text {cluster }}$ is the number of clusters at the beginning of the iteration. ${ }^{15}$ The number of Euclidean distance values to be computed is then

$$
\left(\begin{array}{c}
N_{\mathrm{m}} \\
2
\end{array}\right)+\sum_{i=2}^{N_{\mathrm{it}}}\left(N_{\mathrm{m}}-i\right) \leq\left(N_{\mathrm{m}}-1\right)^{2}
$$

where $N_{\text {it }}$ is the number of iterations (which depends on the measurements and on the threshold $d_{\mathrm{thr}}$ ) and where the upper bound is obtained by observing that $N_{\text {it }} \leq N_{\mathrm{m}}-1$. Thus, the right-hand side of (30) is the number of Euclidean distance computations in case all measurements are clustered together.

In Algorithm 2, the distance between two clusters $\mathscr{Z}_{i}$ and $\mathscr{Z}_{j}$ has been generically denoted by $d_{c}\left(\mathscr{Z}_{i}, \mathscr{Z}_{j}\right)$. With respect to the definition (26) based on cluster centroids, the definition (28) based on the minimum distance between measurements tends to generate a smaller number $N_{\text {cluster }}$ of clusters of relatively larger size, while the definition (29) based on the maximum distance between measurements is more conservative and tends to give rise to a larger number $N_{\text {cluster }}$ of clusters of relatively smaller size.

\section{Object tracking}

The output of the measurement clustering step at scan period $k$ is a finite set of (unordered) measurements $Z_{k}=$ $\left\{\boldsymbol{z}_{1}, \boldsymbol{z}_{2}, \ldots, \boldsymbol{z}_{m_{k}}\right\}_{k}$, where $m_{k}=N_{\text {cluster }}$ and where (with some abuse of notation) $\boldsymbol{z}_{i}$ is the centroid of the $i$ th cluster out of the $N_{\text {cluster }}$ final ones. Individual measurements in the set $Z_{k}$ may be originated both by target detections and by false alarms. An admissible value for $Z_{k}$ is $\emptyset$ (empty set, in case of no false alarms and all targets missed) and its cardinality $m_{k}$ is subject to variations from frame to frame. In the following, the conventional notation $Z^{(k)}$ will be used to indicate the sequence $Z_{1}, Z_{2}, \ldots, Z_{k}$ of all observed measurement sets up to scan period $k$. In the specific case of the LS detector combining CFAR detections from individual sensors, if $Z_{k} \neq \emptyset$ then each measurement $z \in Z_{k}$ comprises position coordinates ( $\boldsymbol{z}=\left[\begin{array}{ll}z_{x} & z_{y}\end{array}\right]^{T}$ in a simple two-dimensional scenario).

The state of a single target will be denoted by $\boldsymbol{x}$ and the single target state space, to which $\boldsymbol{x}$ belongs, by $\mathcal{X}$. The single target state $\boldsymbol{x}$ may in principle include target position, velocity, and acceleration components; in the RSN presented in this paper, sticking to a simple two-dimensional scenario, we have

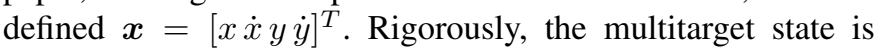
defined as a random finite set (RFS) $X=\left\{\boldsymbol{x}_{1}, \boldsymbol{x}_{2}, \ldots, \boldsymbol{x}_{M}\right\}$ whose cardinality $M$ is the number of targets and whose elements are the states of the $M$ targets. Again, $\emptyset$ (no target) is an admissible value for $X$.

A multitarget Bayesian filter would allow optimally tracking, in every scan period $k$, the a posteriori probability density

\footnotetext{
${ }^{15}$ The number of Euclidean distance values to be computed during all iteration but the first one is not $\left(\begin{array}{c}N_{\text {cluster }} \\ 2\end{array}\right)$ since we only need to compute the distance between the new cluster and the existing ones.
} 
function (p.d.f.) $f_{k \mid k}\left(X \mid Z^{(k)}\right)$ of the multitarget state. ${ }^{16}$ Due to intractable complexity of the theoretically optimum solution, it is common to rely on approximations. A very popular approximation of the multitarget Bayesian filter is represented by Mahler's probability hypothesis density (PHD) filter [145], capable to recursively track the a posteriori multitarget state PHD. This latter quantity, a function $D_{k \mid k}\left(\boldsymbol{x} \mid Z^{(k)}\right): \mathcal{X} \mapsto \mathbb{R}$ (hereafter also abbreviated to $D_{k \mid k}(\boldsymbol{x})$ ), represents the firstorder moment of the a posteriori p.d.f. $f_{k \mid k}\left(X \mid Z^{(k)}\right)$ and is operatively given by

$$
D_{k \mid k}(\boldsymbol{x})=\sum_{n=0}^{\infty} \frac{1}{n !} \int_{\mathcal{X}} f_{k \mid k}\left(\left\{\boldsymbol{x}, \boldsymbol{\xi}_{1}, \ldots, \boldsymbol{\xi}_{n}\right\}\right) \mathrm{d} \boldsymbol{\xi}_{1} \cdots \mathrm{d} \boldsymbol{\xi}_{n} .
$$

It may be interpreted as the density of targets in the single target state space $\mathcal{X}$. As such, its integral $\mathbb{E}[M]_{k \mid k}:=$ $\int_{\mathcal{X}} D_{k \mid k}\left(\boldsymbol{x} \mid Z^{(k)}\right) \mathrm{d} \boldsymbol{x}$ represents the expected number of targets in the scene given the whole measurements history $Z^{(k)}$.

Multitarget state tracking based on the measurements generated by the LS-based detector followed by the clustering step has been performed by employing a basic single-sensor PHD filter (i.e., not in its cardinalized version also tracking the probability mass function of the number of targets in the scene). The single-sensor version of the filter may be employed since, although the sensor radar is composed of several sensors, measurements generation is performed at the fusion center. It should be remarked that the PHD filter derivation (see [145]) is based on a multitarget motion model in which target motions are statistically independent, targets may disappear from the scene, new targets may appear in the scene (independently of existing targets), and new targets may be spawned from existing ones. Furthermore, it assumes a measurement model where each target generates at most one measurement, all measurements are independent conditionally on the multitarget state $X$, missed detections are possible, and the false alarm process is a multi-object Poisson process. While the multitarget motion model seems not critical in UWB sensor radar applications, we underline that fulfillment of the condition of having no more than one measurement per target heavily relies on the success of the described clustering operations.

The basic PHD filter prediction and (approximate) correction equations are

$$
D_{k+1 \mid k}(\boldsymbol{x})=b_{k+1 \mid k}(\boldsymbol{x})+\int_{\mathcal{X}} F_{k+1 \mid k}(\boldsymbol{x} \mid \boldsymbol{\xi}) D_{k \mid k}(\boldsymbol{\xi}) \mathrm{d} \boldsymbol{\xi}
$$

and

$$
\begin{aligned}
D_{k+1 \mid k+1}(\boldsymbol{x}) & \approx D_{k+1 \mid k}(\boldsymbol{x})\left[1-p_{D}(\boldsymbol{x})\right. \\
& \left.+\sum_{\boldsymbol{z} \in Z_{k+1}} \frac{p_{D}(\boldsymbol{x}) \varphi_{k+1}(\boldsymbol{z} \mid \boldsymbol{x})}{\lambda c_{k+1}(\boldsymbol{z})+\int_{\mathcal{X}} p_{D}(\boldsymbol{\xi}) \varphi_{k+1}(\boldsymbol{z} \mid \boldsymbol{\xi}) \mathrm{d} \boldsymbol{\xi}}\right] .
\end{aligned}
$$

\footnotetext{
${ }^{16}$ For a rigorous probabilistic approach to RFSs and multitarget Bayes filtering the reader is referred, for example, to [143] or to [144].
}

In (32), $b_{k+1 \mid k}(\boldsymbol{x})$ is the PHD of new targets appearing in the scene ${ }^{17}$ while $F_{k+1 \mid k}(\boldsymbol{x} \mid \boldsymbol{\xi})=p_{s, k+1 \mid k}(\boldsymbol{\xi}) \mu_{k+1 \mid k}(\boldsymbol{x} \mid \boldsymbol{\xi})+$ $\sigma_{k+1 \mid k}(\boldsymbol{x} \mid \boldsymbol{\xi})$, being $p_{s, k+1 \mid k}(\boldsymbol{\xi})$ the probability that a target existing at time $k$ with state $\boldsymbol{\xi}$ survives at time $k+1$, $\mu_{k+1 \mid k}(\boldsymbol{x} \mid \boldsymbol{\xi})$ the single target motion model (transition density), and $\sigma_{k+1 \mid k}(\boldsymbol{x} \mid \boldsymbol{\xi})$ the PHD of spawning targets. ${ }^{18}$ Furthermore, in (33), $p_{D}(\boldsymbol{x}):=p_{D, k+1}(\boldsymbol{x})$ is the probability that a measurement is collected at scan time $k+1$ from a target having state $\boldsymbol{x}, \lambda:=\lambda_{k+1}$ is the average number of false alarms, $c_{k+1}(\boldsymbol{z})$ is the false alarm distribution in the measurement space, and $\varphi_{k+1}(\boldsymbol{z} \mid \boldsymbol{x})$ is the measurement model (or sensor likelihood function).

Equations (32) and (33) have been implemented using a particle approach. The implemented particle filter comprises the usual steps, i.e., prediction (existing particles at scan time $k$ are updated based on the motion model), correction (particle weights are updated based on new measurements), resampling. New particles are initialized in each iteration around each measurement, in oder to track possible new targets appearing in the scene, the sum of the weights of these newly introduced particles being equal to $\int_{\mathcal{X}} b_{k+1 \mid k}(\boldsymbol{x}) \mathrm{d} \boldsymbol{x}$ as required. The number of particles is maintained constant over time by resampling. At the end of the correction step of each iteration of the particle filter, the particles along with their weights form a discretized version of the PHD $D_{k \mid k}(\boldsymbol{x})$. As such, the sum of all particle weights represents the particle approximation of the (a posteriori) expected number of targets in the scene, $\mathbb{E}[M]_{k \mid k}$. We then define the estimated number of targets at scan time $k, \hat{M}_{k \mid k}$, as the integer closest to $\mathbb{E}[M]_{k \mid k}$ and the (a posteriori) estimated target states as the $\hat{M}_{k \mid k}$ largest peaks of the PHD function. This latter step is performed through an expectation-maximization (EM) algorithm fitting the discretized PHD with $\hat{M}_{k \mid k}$ Gaussian functions.

\section{E. Data association}

In several RSN applications we may want to have one track (sequence of target states) associated with each detected target. When this is the case, a data association problem shall be solved, consisting of performing an association between existing tracks and new filtered measurements. Popular solutions for data association in multi-target tracking problems date back to the Seventies [146]-[149]; another popular technique is the multiple hypothesis tracking (MHT) algorithm [150]. Since a plain PHD filter does not automatically perform data association, this problem has been the subject of several more recent studies in the framework of PHD filtering [151]-[153].

The data association method designed for the proposed sensor radar is a relatively-simple algorithm whose input at scan time $k$ is represented by the set of existing tracks inherited from scan time $k-1$ and by the $\hat{M}_{k \mid k}$ target state estimates (PHD peaks) returned by the PHD filter, and whose output is

\footnotetext{
${ }^{17} b_{k+1 \mid k}(\boldsymbol{x})=\sum_{n=0}^{\infty} \frac{1}{n !} \int_{\mathcal{X}} b_{k+1 \mid k}\left(\left\{\boldsymbol{x}, \boldsymbol{\xi}_{1}, \ldots, \boldsymbol{\xi}_{n}\right\}\right) \mathrm{d} \boldsymbol{\xi}_{1} \cdots \mathrm{d} \boldsymbol{\xi}_{n}$, where $b_{k+1 \mid k}(X)$ is the p.d.f. of new targets with multitarget state $X$ appearing at scan time $k+1$.

${ }^{18} \sigma_{k+1 \mid k}(\boldsymbol{x} \mid \boldsymbol{\xi})=\sum_{n=0}^{\infty} \frac{1}{n !} \int_{\mathcal{X}} \sigma_{k+1 \mid k}\left(\left\{\boldsymbol{x}, \boldsymbol{\xi}_{1}, \ldots, \boldsymbol{\xi}_{n} \mid \boldsymbol{\xi}\right\}\right) \mathrm{d} \boldsymbol{\xi}_{1} \cdots \mathrm{d} \boldsymbol{\xi}_{n}$, where $\sigma_{k+1 \mid k}(X \mid \boldsymbol{\xi})$ is the p.d.f. of new targets with multitarget state $X$ spawned at scan time $k+1$ from a target with state $\boldsymbol{\xi}$ at time $k$.
} 
an updated set of tracks $\mathcal{T}_{k}$ (see Fig. 1). Thus, in the proposed system data association is performed after PHD filtering.

The algorithm may be summarized as follows. At each scan, a cost is calculated for each hypothesis, where an hypothesis is an association between the $\hat{M}_{k \mid k}$ state estimates and the existing tracks. The hypothesis with minimum cost is then considered. If its cost is lower than a maximum acceptable cost, then the association is valid: a state estimate associated with an existing track yields an updated track, a track not associated with any state estimate is declared to be silent, an estimated state not associated with any existing track yields the initialization of a new track. ${ }^{19}$ In contrast, if the cost of the minimum-cost hypothesis is larger than the maximum acceptable value, a gating procedure is started in which each association foreseen by the minimum cost hypothesis is individually considered: the single association is declared to be valid if and only if the estimated state belongs to a properlydefined permitted region around the track head. For all single associations declared to be invalid, the corresponding track is declared to be silent, and a new track is initiated for the corresponding estimated state.

A state is defined and updated for each track. Possible track states are unconfirmed, confirmed, silent, dead; the set $\mathcal{T}_{k}$ of existing tracks only comprises unconfirmed, confirmed, and silent ones. The state of every newly initiated track is unconfirmed. It is turned to confirmed after a certain number of scans, in all of which the track has been updated. The state of a confirmed track for which, at some scan, no valid association with any PHD-estimated state is found, is turned to silent. If a valid association is found between a target state and a silent track within a certain number of scan periods, the track state is reverted back to confirmed. Otherwise the silent track state is turned to dead and the track is removed from the set of existing tracks. (The silent state has been introduced to cope with detected targets that suddenly stop their motion.) An unconfirmed track, for which no valid association is found in some scan before the track is declared confirmed, is directly turned to a dead track. This way, we can cope with sporadic false alarms not filtered out by the PHD filter.

\section{A CASE STUdy}

A measurement campaign has been carried out for a single target in an indoor scenario with a RSN composed of a Tx and $N_{\mathrm{r}}=6 \mathrm{Rxs}$ placed at the sides of a rectangular area of size $11.5 \mathrm{~m} \times 6 \mathrm{~m}$. A sketch of the environment with the monitored area indicated in pink is provided in Fig. 2, and the corresponding sensor node locations are depicted in Fig. 7.

The sensors adopted to transmit and collect UWB waveforms are the Time Domain's PulsOn 410 [154]. Such transceivers are designed for short range, low cost and relatively low power applications. Each node has two antenna ports, a power interface, and a port for data transmission and control. The sensors are connected with a personal computer to control the transmission and reception parameters as well

\footnotetext{
${ }^{19}$ In fact, the number $\hat{M}_{k \mid k}$ may be equal to, lower than, or larger than the number of existing tracks from scan time $k-1$.
}

as to collect all the received waveforms (the vector $\mathbf{r}$ in Section IV-A) through the Channel Analysis Tool (CAT) software [154].

The PSD of the transmitted signal is centered at frequency $f_{\mathrm{c}}=4.3 \mathrm{GHz}$ with bandwidth $B=2.2 \mathrm{GHz}$, the transmit power has been set to $P_{\mathrm{t}}=-12.64 \mathrm{dBm}$, and the receiver noise figure is $4.8 \mathrm{~dB}$. The antennas adopted in the experimental campaign are UWB omnidirectional dipoles with approximately $0 \mathrm{~dB}$ gain. The human target, of $90 \mathrm{Kg}$ weight and $185 \mathrm{~cm}$ height, moves with uniform linear motion (constant velocity) from right to left along the blue trajectory depicted in Fig. 7, with speed $1.5 \mathrm{~m} / \mathrm{s}$. The trajectory is $9.15 \mathrm{~m}$ long corresponding to $N_{\text {scan }}=61$ scans, thus the spatial resolution is $15 \mathrm{~cm} / \mathrm{scan}$.

With reference to the LS detector described in Sec. V-A, the value $\nu=3$ has been used for measurements generation. To decrease the false alarm rate as proposed in Sec. V-B, different measurement weight thresholds, $\Omega_{\text {th }}$, and RE thresholds, $\mathrm{RE}_{\mathrm{th}}$, are considered. In particular, the target track has been estimated with three parameter settings: A) $\Omega_{\text {th }}=40$, $\left.\left.\mathrm{RE}_{\mathrm{th}}=6 \cdot 10^{-13} \mathrm{~m}^{3} ; \mathrm{B}\right) \Omega_{\mathrm{th}}=56, \mathrm{RE}_{\mathrm{th}}=4 \cdot 10^{-13} \mathrm{~m}^{3} ; \mathrm{C}\right)$ $\Omega_{\mathrm{th}}=56, \mathrm{RE}_{\mathrm{th}}=6 \cdot 10^{-13} \mathrm{~m}^{3}$. The remaining parameters of the RSN can be found in Sec. IV.

The localization error is defined as the distance between the true position of the target, $\mathbf{p}_{k}=\left(x_{k}, y_{k}\right)$, and the estimated one, $\hat{\mathbf{p}}_{k}=\left(\hat{x}_{k}, \hat{y}_{k}\right)$, in the $k$ th scan, i.e., $\left\|\mathbf{p}_{k}-\hat{\mathbf{p}}_{k}\right\|$. The related root mean-square error (RMSE) is

$$
\mathrm{RMSE}=\sqrt{\frac{1}{N_{\text {scan }}} \sum_{k=1}^{N_{\text {scan }}}\left\|\mathbf{p}_{k}-\hat{\mathbf{p}}_{k}\right\|^{2}}
$$

and the empirical cumulative distribution function (c.d.f.) is

$$
\mathcal{F}(\varepsilon)=\frac{1}{N_{\text {scan }}} \sum_{k=1}^{N_{\text {scan }}} \mathbb{1}_{\left\|\mathbf{p}_{k}-\hat{\mathbf{p}}_{k}\right\| \leqslant \varepsilon}
$$

where $\mathbb{1}_{\mathcal{A}}$ is the indicator function, i.e., $\mathbb{1}_{\mathcal{A}}=1$ when $\mathcal{A}$ is true and 0 otherwise.

The estimated tracks for the three parameter settings A, B, and $\mathrm{C}$, after the whole processing, are depicted in Fig. 7, and the corresponding empirical c.d.f.s of the localization error are shown in Fig. 8. As can be seen, the localization error is less than $40 \mathrm{~cm}$ and $60 \mathrm{~cm}$ in almost $80 \%$ of the target trajectory for the tracks $\mathrm{A}$ and $\mathrm{C}$, respectively. The RMSE is $36 \mathrm{~cm}$ for track A, $61 \mathrm{~cm}$ for track B, and $54 \mathrm{~cm}$ for track C. It is worth noting that such error is less than the human target size, hence it is relatively small.

Interestingly, track A in Fig. 7 is the one that appears less smooth despite its RMSE is the lowest among the three tracks. On the contrary, tracks B and C appear less spiky although their empirical c.d.f.s are below that of track A. This behavior is due in part to the fact that the localization error can occur also along the target trajectory, a situation which is not visible in the plots of Fig. 7. This trade-off between accuracy and smoothness can be exploited by the system designer to choose the proper set of parameters to meet specific application requirements for the RSN. 


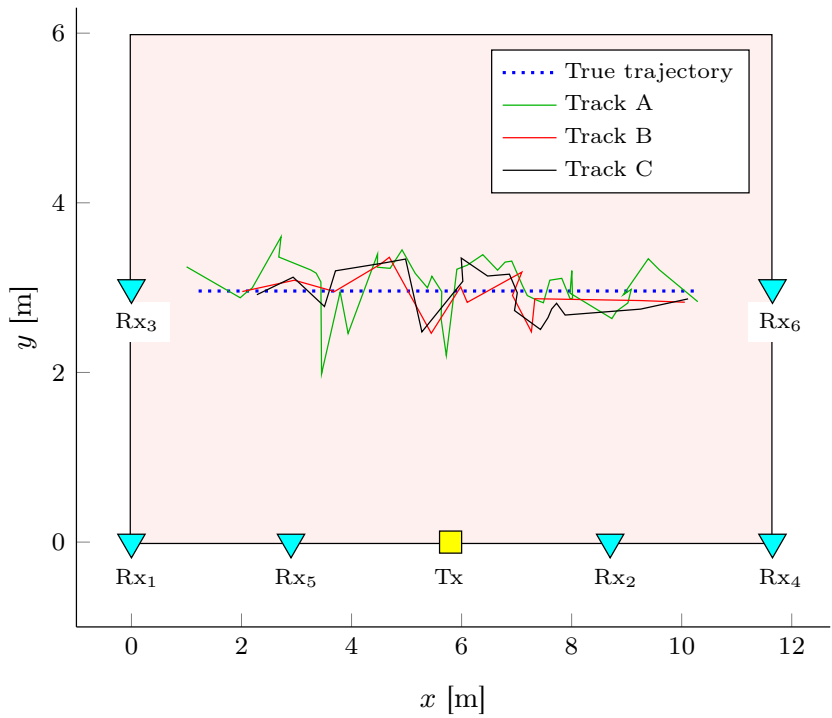

Fig. 7. The RSN configuration in the case study scenario depicted in Fig. 2, the trajectory of the target, and the estimated tracks for different parameter settings: A (green), B (red) and C (black). The monitored area is represented as in Fig. 2 with the pink rectangle.

\section{CONCLUSIONS AND FinAl REMARKS}

This paper presented the fundamental aspects of noncollaborative object localization in indoor environments using IR-UWB technology. To better understand the steps that lead to object tracking, a RSN demonstrator has been used to illustrate the signals and data processed, when operating in a real indoor scenario. The case study assessed that the localization accuracy level that can be reached is very promising and in the order of tens of centimeters.

Ubiquitous deployment of sensor radar systems is expected to open new research directions and challenges. Examples are represented by reliably tracking a large number of objects in densely cluttered areas, the design of low-complexity architectures, real-time capabilities, and energy efficient in-sensor processing design.

Concerning the specific steps described in this paper, it has been highlighted how the first network processing step (measurement generation, Sec. V-A) has the disadvantage to give rise to a considerable number of false alarms, which must then be carefully filtered out by a false alarm mitigation strategy (based on both measurement "weight" and RE) and subsequently by measurement clustering. Although the effectiveness of these strategies has been verified with actual measurements, the overall network processing would benefit from a lower number of false alarms in the detection step. In this respect, it should be observed that different Rx nodes generally experience different channel qualities with respect to different targets and that performing a target-specific sensor node ranking becomes feasible if a feedback from the tracking filter is available. This is expected to enable the generation of measurements for targets being tracked using only few selected sensor nodes, leading to a reduced number of false alarms and a lower computational burden on the false alarm mitigation steps.

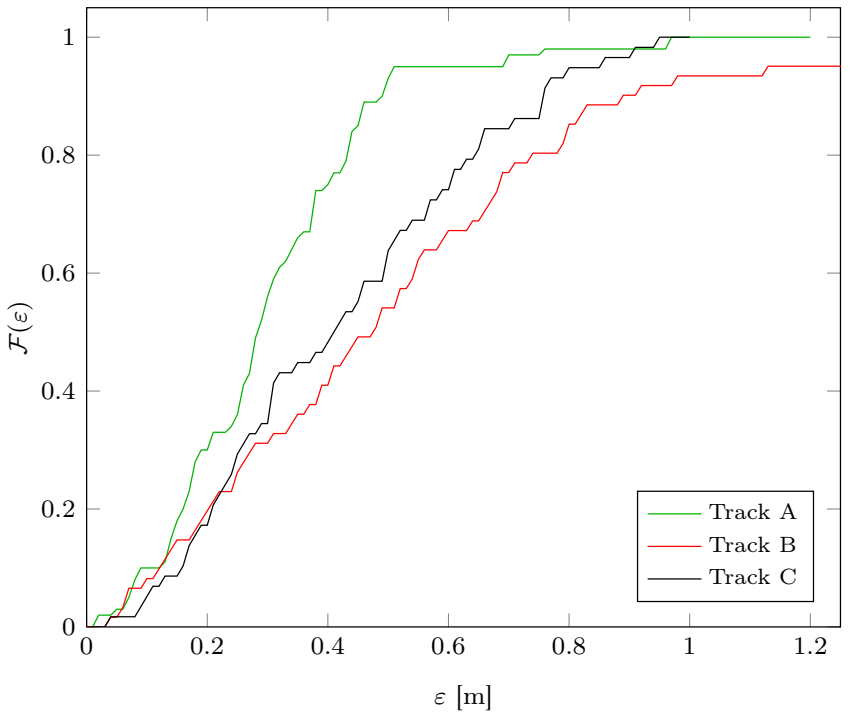

Fig. 8. The empirical c.d.f. of the localization error of the estimated tracks for different parameter settings: A (green), B (red) and C (black).

Readers may have noted that Doppler effect is not exploited by the described radar sensor network. This choice has been dictated by the need to develop a low-complexity processing and by challenges in effectively exploiting Doppler effect when using UWB waveforms to detect targets having similar velocity (as it is often the case in indoor applications). Anyhow, a judicious processing allows extracting Doppler information also in this challenging context (e.g., [155], [156]), which opens interesting directions of investigations, for example, concerning classification of detected targets that are being tracked by the sensor radar.

Readers acquainted with multi-object tracking may also wonder if a cardinalized PHD (CPHD) filter [157] or a multiBernoulli filter [158]-[160] may be able to improve the sensor radar performance. The use of a CPHD filter (propagating the random set cardinality distribution and its probability generating function along with the PHD) is expected to improve performance, but at the cost of additional computational complexity. Multi-Bernoulli filters, which propagate the parameters of a multi-Bernoulli distribution approximating the a posteriori p.d.f. of the multitarget state instead of its firstorder moment, are characterized by a complexity similar to that of the PHD filter. Their suitability with respect to the measurement generated according to the proposed processing should be carefully evaluated.

\section{ACKNOWLEDGMENT}

The authors would like to thank Matteo Mazzotti, Bita Sobhani, and Mauro Montanari, for helpful discussions, as well as the anonymous reviewers for their insightful comments.

\section{REFERENCES}

[1] M. Z. Win, A. Conti, S. Mazuelas, Y. Shen, W. M. Gifford, D. Dardari, and M. Chiani, "Network localization and navigation via cooperation," IEEE Commun. Mag., vol. 49, no. 5, pp. 56-62, May 2011. 
[2] N. Patwari, J. Ash, S. Kyperountas, I. Hero, A.O., R. Moses, and N. Correal, "Locating the nodes: cooperative localization in wireless sensor networks," IEEE Signal Process. Mag., vol. 22, no. 4, pp. 54-69, Jul. 2005.

[3] E. A. de Reyna and P. M. Djuric, "Indoor localization with range-based measurements and little prior information," IEEE Sensors J., vol. 13, no. 5, pp. 1979-1987, May 2013.

[4] Y. Shen and M. Z. Win, "Fundamental limits of wideband localization Part I: A general framework," IEEE Trans. Inf. Theory, vol. 56, no. 10, pp. 4956-4980, Oct. 2010.

[5] Y. Shen, H. Wymeersch, and M. Z. Win, "Fundamental limits of wideband localization - Part II: Cooperative networks," IEEE Trans. Inf. Theory, vol. 56, no. 10, pp. 4981-5000, Oct. 2010.

[6] Y. Shen, S. Mazuelas, and M. Z. Win, "Network navigation: Theory and interpretation," IEEE J. Sel. Areas Commun., vol. 30, no. 9, pp. 1823-1834, Oct. 2012

[7] P. Withington, H. Fluhler, and S. Nag, "Enhancing homeland security with advanced UWB sensors," IEEE Microw. Mag., vol. 4, no. 3, pp. 51-58, Sep. 2003.

[8] E. Cianca, M. De Sanctis, and S. Di Domenico, "Radios as sensors," IEEE Internet Things J., vol. 4, no. 2, pp. 363-373, Apr. 2017.

[9] B. Beck, R. Baxley, and J. Kim, "Real-time, anchor-free node tracking using ultrawideband range and odometry data," in IEEE Int. Conf. on Ultra-WideBand (ICUWB), Paris, France, Sep. 2014, pp. 286-291.

[10] M. Bocca, O. Kaltiokallio, N. Patwari, and S. Venkatasubramanian, "Multiple target tracking with RF sensor networks," IEEE Trans. Mobile Comput., vol. 13, no. 8, pp. 1787-1800, Jul. 2014.

[11] A. Giorgetti, M. Lucchi, E. Tavelli, M. Barla, G. Gigli, N. Casagli, M. Chiani, and D. Dardari, "A robust wireless sensor network for landslide risk analysis: System design, deployment, and field testing," IEEE Sensors J., vol. 16, no. 16, pp. 6374-6386, Aug. 2016.

[12] A. Conti, M. Guerra, D. Dardari, N. Decarli, and M. Z. Win, "Network experimentation for cooperative localization," IEEE J. Sel. Areas Commun., vol. 30, no. 2, pp. 467-475, Feb. 2012.

[13] S. Mazuelas, Y. Shen, and M. Z. Win, "Belief condensation filtering," IEEE Trans. Signal Process., vol. 61, no. 18, pp. 4403-4415, Sep. 2013.

[14] A. Conti, D. Dardari, M. Guerra, L. Mucchi, and M. Z. Win, "Experimental characterization of diversity navigation," IEEE Syst. J., vol. 8, no. 1, pp. 115-124, Mar. 2014.

[15] W. W.-L. Li, Y. Shen, Y. J. Zhang, and M. Z. Win, "Robust power allocation for energy-efficient location-aware networks," IEEE/ACM Trans. Netw., vol. 21, no. 6, pp. 1918-1930, Dec. 2013.

[16] Y. Shen, W. Dai, and M. Z. Win, "Power optimization for network localization," IEEE/ACM Trans. Netw., vol. 22, no. 4, pp. 1337-1350, Aug. 2014.

[17] T. V. Nguyen, Y. Jeong, H. Shin, and M. Z. Win, "Least-square cooperative localization," IEEE Trans. Veh. Technol., vol. 64, no. 4, pp. 1318-1330, Apr. 2015.

[18] A. Conti, J. Wang, H. Shin, R. Annavajjala, and M. Z. Win, "Wireless cooperative networks," EURASIP J. Appl. Signal Process., vol. 2008, pp. 1-2, Article ID 810149,2008 , special issue on Wireless Cooperative Networks.

[19] D. Dardari, A. Conti, J. Lien, and M. Z. Win, "The effect of cooperation on localization systems using UWB experimental data," EURASIP J. Appl. Signal Process., vol. 2008, pp. 1-11, Article ID 513873, 2008, special issue on Cooperative Localization in Wireless Ad Hoc and Sensor Networks.

[20] Y. Shen and M. Z. Win, "On the accuracy of localization systems using wideband antenna arrays," IEEE Trans. Commun., vol. 58, no. 1, pp. 270-280, Jan. 2010

[21] S. Maranò, W. M. Gifford, H. Wymeersch, and M. Z. Win, "NLOS identification and mitigation for localization based on UWB experimental data," IEEE J. Sel. Areas Commun., vol. 28, no. 7, pp. 1026-1035, Sep. 2010.

[22] W. C. Headley, C. R. C. M. da Silva, and R. M. Buehrer, "Indoor location positioning of non-active objects using ultra-wideband radios," in IEEE Radio and Wireless Symp. (RWS), Long Beach, CA, USA, Jan. 2007, pp. 105-108.

[23] F. Colone, P. Falcone, C. Bongioanni, and P. Lombardo, "WiFibased passive bistatic radar: data processing schemes and experimental results," IEEE Trans. Aerosp. Electron. Syst., vol. 48, no. 2, pp. 10611079, Apr. 2012.

[24] P. Falcone, F. Colone, and P. Lombardo, "Potentialities and challenges of WiFi-based passive radar," IEEE Aerosp. Electron. Syst. Mag., vol. 27 , no. 11 , pp. 15-26, Nov. 2012.
[25] F. Colone, D. Pastina, P. Falcone, and P. Lombardo, "WiFi-based passive ISAR for high-resolution cross-range profiling of moving targets," IEEE Trans. Geosci. Remote Sens., vol. 52, no. 6, pp. 34863501, Jun. 2014

[26] M. I. Skolnik, Radar Handbook, 2nd ed. McGraw-Hill Professional, Jan. 1990.

[27] V. S. Chernyak, Fundamentals of Multisite Radar Systems. Gordon and Breach Science Publisher, 1998.

[28] "IEEE Standard Radar Definitions," IEEE Std 686-2008 (Revision of IEEE Std 686-1997), pp. c1-41, May 2008.

[29] "IEEE Standard for Ultrawideband Radar Definitions," IEEE Std 1672 2006, pp. c1-9, May 2007.

[30] X. Gong, J. Zhang, D. Cochran, and K. Xing, "Optimal placement for barrier coverage in bistatic radar sensor networks," IEEE/ACM Trans. Netw., vol. 24, no. 1, pp. 259-271, Feb. 2016.

[31] F. C. Robey, S. Coutts, D. Weikle, J. C. McHarg, and K. Cuomo, "MIMO radar theory and experimental results," in Asilomar Conf. on Signals, Systems and Comp., vol. 1, Pacific Grove, CA, USA, Nov. 2004, pp. 300-304.

[32] A. M. Haimovich, R. S. Blum, and L. J. Cimini, "MIMO radar with widely separated antennas," IEEE Signal Process. Mag., vol. 25, no. 1, pp. 116-129, Jan. 2008

[33] E. Brookner, "MIMO radar demystified and where it makes sense to use," in Int. Radar Conf. (Radar), Lille, France, Oct. 2014, pp. 1-6.

[34] D. Hong, C. Chen, P. Shirui, L. Xin, and Z. Linhua, "Multistatic ultra-wideband localization for NLOS environments," in Int. Conf. on Intelligent Sys. Design and Eng. App. (ISDEA), Sanya, Hainan, China, Jan. 2012, pp. 380-384.

[35] S. Bartoletti, A. Giorgetti, and A. Conti, "Sensor radars with subset diversity," in Proc. IEEE Int. Conf. on Commun. (ICC), Work. on Adv in Net. Localization and Navigation (ANLN), Budapest, Hungary, Jun. 2013, pp. 32-36.

[36] S. Bartoletti, A. Conti, A. Giorgetti, and M. Z. Win, "Sensor radar networks for indoor tracking," IEEE Wireless Commun. Lett., vol. 3 , no. 2, pp. 157-160, Apr. 2014

[37] S. Bartoletti, A. Giorgetti, M. Z. Win, and A. Conti, "Blind selection of representative observations for sensor radar networks," IEEE Trans. Veh. Technol., vol. 64, no. 4, pp. 1388-1400, Apr. 2015.

[38] M. Z. Win and R. A. Scholtz, "Impulse radio: How it works," IEEE Commun. Lett., vol. 2, no. 2, pp. 36-38, Feb. 1998.

[39] _ _ "Ultra-wide bandwidth time-hopping spread-spectrum impulse radio for wireless multiple-access communications," IEEE Trans. Commun., vol. 48, no. 4, pp. 679-691, Apr. 2000.

[40] — "Characterization of ultra-wide bandwidth wireless indoor communications channel: A communication-theoretic view," IEEE J. Sel. Areas Commun., vol. 20, no. 9, pp. 1613-1627, Dec. 2002.

[41] P. A. Catherwood and W. G. Scanlon, "Ultrawideband communications - an idea whose time has still yet to come?" IEEE Antennas Propag. Mag., vol. 57, no. 2, pp. 38-43, 2015.

[42] W. Suwansantisuk, M. Z. Win, and L. A. Shepp, "On the performance of wide-bandwidth signal acquisition in dense multipath channels," IEEE Trans. Veh. Technol., vol. 54, no. 5, pp. 1584-1594, Sep. 2005.

[43] M. Z. Win, G. Chrisikos, and A. F. Molisch, "Wideband diversity in multipath channels with nonuniform power dispersion profiles," IEEE Trans. Wireless Commun., vol. 5, no. 5, pp. 1014-1022, May 2006.

[44] M. Z. Win, D. Dardari, A. F. Molisch, W. Wiesbeck, and J. Zhang, "History and applications of UWB," Proc. IEEE, vol. 97, no. 2, pp. 198-204, Feb. 2009

[45] N. Decarli, A. Giorgetti, D. Dardari, M. Chiani, and M. Z. Win, "Stopand-Go receivers for non-coherent impulse communications," IEEE Trans. Wireless Commun., vol. 13, no. 9, pp. 4821-4835, Sep. 2014.

[46] S. Gezici, T. Zhi, G. Giannakis, H. Kobayashi, A. Molisch, H. Poor, and Z. Sahinoglu, "Localization via ultra-wideband radios: a look at positioning aspects for future sensor networks," IEEE Signal Process. Mag., vol. 22, no. 4, pp. 70-84, Jul. 2005.

[47] D. Dardari, A. Conti, U. Ferner, A. Giorgetti, and M. Z. Win, "Ranging with ultrawide bandwidth signals in multipath environments," Proc. IEEE, vol. 97, no. 2, pp. 404-426, Feb. 2009, Invited Paper, (Special Issue on UWB Technology and Emerging Applications).

[48] R. Zekavat and R. Buehrer, Wireless Localization Using UltraWideband Signals, 1st ed. Wiley-IEEE Press, 2012.

[49] A. Giorgetti and M. Chiani, "Time-of-arrival estimation based on information theoretic criteria," IEEE Trans. Signal Process., vol. 61, no. 8, pp. 1869-1879, Apr. 2013.

[50] A. Giorgetti, M. Chiani, and M. Z. Win, "The effect of narrowband interference on wideband wireless communication systems," IEEE Trans. Commun., vol. 53, no. 12, pp. 2139-2149, Dec. 2005. 
[51] M. Chiani and A. Giorgetti, "Coexistence between UWB and narrowband wireless communication systems," Proc. IEEE, vol. 97, no. 2, pp. 231-254, Feb. 2009, Invited Paper, (Special Issue on UWB Technology and Emerging Applications).

[52] P. Pinto, A. Giorgetti, M. Z. Win, and M. Chiani, "A stochastic geometry approach to coexistence in heterogeneous wireless networks," IEEE J. Sel. Areas Commun., vol. 27, no. 7, pp. 1268-1282, Sep. 2009, (Special Issue on Stochastic Geometry and Random Graphs for Wireless Networks).

[53] M. G. M. Hussain, "Ultra-wideband impulse radar - an overview of the principles," IEEE Aerosp. Electron. Syst. Mag., vol. 13, no. 9, pp. 9-14, Sep. 1998.

[54] V. Chernyak, "Multi-site ultra-wideband radar systems with information fusion: some principal features," in Int. Conf. on Information Fusion (FUSION), Stockholm, Sweden, Jun. 2004, pp. 463-470.

[55] R. Thoma, O. Hirsch, J. Sachs, and R. Zetik, "UWB sensor networks for position location and imaging of objects and environments," in Europ. Conf. Ant. and Prop. (EuCAP), Edinburgh, UK, Nov. 2007, pp. $1-9$.

[56] W. Guohua, Z. Yuxiang, and W. Siliang, "Detection and localization of high speed moving targets using a short-range UWB impulse radar," in IEEE Radar Conf., Rome, Italy, May 2008, pp. 1-4.

[57] E. Paolini, A. Giorgetti, M. Chiani, R. Minutolo, and M. Montanari, "Localization capability of cooperative anti-intruder radar systems," EURASIP J. Adv. in Signal Proc., vol. 2008, pp. 1-14, Apr. 2008.

[58] I. Y. Immoreev, "Ultrawideband radars: Features and capabilities," $J$. Commun. Technol. Electron., vol. 54, no. 1, pp. 1-26, Feb. 2009.

[59] J. Liang and Q. Liang, "Design and analysis of distributed radar sensor networks," IEEE Trans. Parallel Distrib. Syst., vol. 22, no. 11, pp. 1926-1933, Nov. 2011.

[60] D. Kocur, D. Novak, and J. Rovňáková, "Moving person tracking by UWB radar system in complex environment," in IEEE Int. Symp. Intell. Signal Proc. (WISP), Funchal, Portugal, Sep. 2013, pp. 77-82.

[61] D. Kocur, P. Kažimír, J. Fortes, D. Novák, M. Drutarovskỳ, P. Galajda, and R. Zetik, "Short-range UWB radar: Surveillance robot equipment of the future," in IEEE Int. Conf. on Systems, Man and Cybernetics (SMC), San Diego, CA, USA, Oct. 2014, pp. 3767-3772.

[62] T. Sakamoto, T. Sato, P. Aubry, and A. Yarovoy, "Fast and accurate UWB radar imaging using hybrid of kirchhoff migration and Stolt's FK migration with inverse boundary scattering transform," in IEEE Int. Conf. on Ultra-WideBand (ICUWB), Paris, France, Sep. 2014, pp. 191-196.

[63] W. Khawaja, K. Sasaoka, and I. Guvenc, "UWB radar for indoor detection and ranging of moving objects: An experimental study," in Int. Work. on Antenna Tech. (iWAT), Cocoa Beach, FL, USA, Feb. 2016, pp. 102-105.

[64] I. Maherin and Q. Liang, "Multistep information fusion for target detection using UWB radar sensor network," IEEE Sensors J., vol. 15, no. 10, pp. 5927-5937, Oct. 2015.

[65] F. Valmori, A. Giorgetti, M. Mazzotti, E. Paolini, and M. Chiani, "Indoor detection and tracking of human targets with UWB radar sensor networks," in IEEE Int. Conf. on Ubiquitous Wireless Broadband (ICUWB), Nanjing, China, Oct. 2016, pp. 1-4.

[66] A. Yarovoy, L. Ligthart, J. Matuzas, and B. Levitas, "UWB radar for human being detection," IEEE Aerosp. Electron. Syst. Mag., vol. 21, no. 3, pp. 10-14, Apr. 2006.

[67] M. Aftanas, J. Rovnáková, M. Drutarovsky, and D. Kocur, "Efficient method of TOA estimation for through wall imaging by UWB radar," in IEEE Int. Conf. on Ultra-Wideband (ICUWB), vol. 2, Hannover, Germany, Sep. 2008, pp. 101-104.

[68] D. Kocur, J. Rovňáková, and M. Švecová, "Through wall tracking of moving targets by M-sequence UWB radar," in Towards Intelligent Engineering and Inf. Tech. Springer, 2009, pp. 349-364.

[69] D. Kocur, J. Gamec, M. Švecová, M. Gamcova, and J. Rovňáková, "Imaging method: A strong tool for moving target tracking by a multistatic UWB radar system," in IEEE Int. Symp. Applied Machine Intelligence and Inf. (SAMI), Herlany, Slovakia, Jan. 2010, pp. 11-19.

[70] Z. Xiao-wei, A. Gaugue, C. Liebe, J. Khamlichi, and M. Ménard, "Through the wall detection and localization of a moving target with a bistatic UWB radar system," in European Radar Conf. (EuRAD), Paris, France, Sep. 2010, pp. 204-207.

[71] T. Kai, W. Shiyou, C. Jie, L. Xiaojun, and F. Guangyou, "An efficient and low-complexity through wall moving target tracking algorithm by UWB radar," in Int. Conf. Ground Penetrating Radar (GPR), Shanghai, China, Jun. 2012, pp. 966-971.
[72] J. Li, Z. Zeng, J. Sun, and F. Liu, "Through-wall detection of human being's movement by UWB radar," IEEE Geosci. Remote Sens. Lett., vol. 9, no. 6, pp. 1079-1083, Nov. 2012.

[73] M. G. Amin, Through-the-wall radar imaging. CRC press, 2016.

[74] D. Dardari, R. D'Errico, C. Roblin, A. Sibille, and M. Z. Win, "Ultrawide bandwidth RFID: The next generation?" Proc. IEEE, vol. 98, no. 9, pp. 1570-1582, Sep. 2010.

[75] N. Decarli, F. Guidi, and D. Dardari, "A novel joint RFID and radar sensor network for passive localization: Design and performance bounds," IEEE J. Sel. Topics Signal Process., vol. 8, no. 1, pp. 80-95, Feb. 2014.

[76] — - "Passive UWB RFID for tag localization: Architectures and design," IEEE Sensors J., vol. 16, no. 5, pp. 1385-1397, Mar. 2016.

[77] V. Chernyak, "Signal processing in multisite UWB radar devices for searching survivors in rubble," in European Radar Conf. (EuRAD), Manchester, UK, Sep. 2006, pp. 190-193.

[78] A. Nezirovic, A. G. Yarovoy, and L. P. Ligthart, "Signal processing for improved detection of trapped victims using UWB radar," IEEE Trans. Geosci. Remote Sens., vol. 48, no. 4, pp. 2005-2014, 2010.

[79] J. Rovňáková and D. Kocur, "Short range tracking of moving persons by UWB sensor network," in European Radar Conf. (EuRAD), Manchester, UK, Oct. 2011, pp. 321-324.

[80] Z. Zhang, X. Zhang, H. Lv, G. Lu, X. Jing, and J. Wang, "Humantarget detection and surrounding structure estimation under a simulated rubble via UWB radar," IEEE Geosci. Remote Sens. Lett., vol. 10, no. 2, pp. 328-331, Mar. 2013.

[81] J. Li, L. Liu, Z. Zeng, and F. Liu, "Advanced signal processing for vital sign extraction with applications in UWB radar detection of trapped victims in complex environments," IEEE J. Sel. Topics Appl. Earth Observ. Remote Sens., vol. 7, no. 3, pp. 783-791, Mar. 2014.

[82] E. Cianca and B. Gupta, "FM-UWB for communications and radar in medical applications," Wireless Personal Comm., vol. 51, no. 4, pp. 793-809, Jul. 2009.

[83] D. Novák, D. Kocur, and J. Demčák, "Static person detection and localization with estimation of person's breathing rate using single multistatic UWB radar," in IEEE Int. Symp. Applied Machine Intelligence and Inf. (SAMI), Herlany, Slovakia, Jan. 2017, pp. 143-148.

[84] E. Ricci, E. Cianca, and T. Rossi, "Beamforming algorithms for UWB radar-based stroke detection: Trade-off performance-complexity," $J$. Comm., Nav., Sens. Serv., vol. 2016, no. 1, pp. 11-28, Jan. 2016.

[85] J. W. Choi, S. H. Cho, Y. S. Kim, N. J. Kim, S. S. Kwon, and J. S. Shim, "A counting sensor for inbound and outbound people using IR-UWB radar sensors," in IEEE Sensors Applications Symp. (SAS), Catania, Italy, Apr. 2016, pp. 1-5.

[86] R. Salman, I. Willms, T. Sakamoto, T. Sato, and A. Yarovoy, "Environmental imaging with a mobile UWB security robot for indoor localisation and positioning applications," in European Radar Conf. (EuRAD), Nuremberg, Germany, Oct. 2013, pp. 331-334.

[87] T. Sakamoto, T. Sato, P. Aubry, and A. Yarovoy, "Fast imaging method for security systems using ultrawideband radar," IEEE Trans. Aerosp. Electron. Syst., vol. 52, no. 2, pp. 658-670, Apr. 2016.

[88] S. Gubinelli, E. Paolini, A. Giorgetti, M. Mazzotti, A. Rizzo, E. Troiani, and M. Chiani, "An ultra-wideband radar approach to nondestructive testing," in IEEE Int. Conf. Ultra-Wideband (ICUWB), Paris, France, Sep. 2014, pp. 303-308.

[89] T. Sakamoto, T. Sato, P. J. Aubry, and A. G. Yarovoy, "Texture-based automatic separation of echoes from distributed moving targets in UWB radar signals," IEEE Trans. Geosci. Remote Sens., vol. 53, no. 1, pp. 352-361, 2015.

[90] Q. Liang, "Radar sensor wireless channel modeling in foliage environment: UWB versus narrowband," IEEE Sensors J., vol. 11, no. 6, pp. 1448-1457, Jun. 2011.

[91] M. Chiani, A. Giorgetti, M. Mazzotti, R. Minutolo, and E. Paolini, "Target detection metrics and tracking for UWB radar sensor networks," in IEEE Int. Conf. Ultra-Wideband (ICUWB), Vancouver, CANADA, Sep. 2009, pp. 469-474.

[92] Y. He, T. Savelyev, and A. Yarovoy, "Two-stage algorithm for extended target tracking by multistatic UWB radar," in IEEE CIE Int. Conf. on Radar, vol. 1, Chengdu, China, Oct. 2011, pp. 795-799.

[93] K. Dae-Hyun, L. Dong-Woo, S. L. K. Hyung-Myung, W.Sung-Chul, and K. Hyun, "Localization methods of multi-targets for UWB radar sensor networks," in Int. Asia-Pac. Conf. on Synth. Aperture Radar (APSAR), Seoul, Korea (South), Sep. 2011, pp. 1-4.

[94] M. McCracken and N. Patwari, "Hidden markov estimation of bistatic range from cluttered ultra-wideband impulse responses," IEEE Trans. Mobile Comput., vol. 13, no. 7, pp. 1509-1521, Jul. 2014. 
[95] S. A. T. Hosseini, H. Amindavar, and J. A. Ritcey, "Robust detection in ultra-wideband impulse radar using DPSS-MMSE estimator,' EURASIP J. Adv. Signal Process., vol. 2016, no. 1, pp. 1-15, Dec. 2016.

[96] Q. Liang, "Biologically inspired target recognition in radar sensor networks," EURASIP J. on Wireless Comm. and Netw., no. 1, pp. 523435, Dec. 2009

[97] T. Thiasiriphet and J. Lindner, "Particle filtering for uwb radar applications," in IEEE Int. Conf. Ultra-Wideband (ICUWB), Bologna, Italy, Sep. 2011, pp. 248-252.

[98] S. Bartoletti, A. Giorgetti, and A. Conti, "UWB sensor radar networks for indoor passive navigation," in Proc. Tyrrhenian Work. on Advances in Radar and Remote Sensing, Napoli, Italy, Sep. 2012, pp. 1-6.

[99] B. Sobhani, E. Paolini, A. Giorgetti, M. Mazzotti, and M. Chiani, "Bayesian tracking in UWB radar sensor networks," in Proc. IEEE Int. Conf. on Commun. (ICC), Work. on Adv. in Net. Localization and Navigation (ANLN), Budapest, Hungary, Jun. 2013, pp. 47-51.

[100] B. Sobhani, M. Mazzotti, E. Paolini, A. Giorgetti, and M. Chiani, "Effect of state space partitioning on bayesian tracking for UWB radar sensor networks," in IEEE Int. Conf. Ultra-Wideband (ICUWB), Sydney, Australia, Sep. 2013, pp. 120-125

[101] B. Gülmezoğlu, "Indoor multi-person tracking via ultra-wideband radars," Ph.D. dissertation, Bilkent University, 2014.

[102] B. Sobhani, M. Mazzotti, E. Paolini, A. Giorgetti, and M. Chiani, "Multiple target detection and localization in UWB multistatic radars,' in IEEE Int. Conf. Ultra-Wideband (ICUWB), Paris, France, Sep. 2014.

[103] B. Sobhani, E. Paolini, A. Giorgetti, M. Mazzotti, and M. Chiani, "Target tracking for UWB multistatic radar sensor networks," IEEE J. Sel. Topics Signal Process., vol. 8, no. 1, pp. 125-136, Feb. 2014.

[104] B.-H. Kim, S.-J. Han, G.-R. Kwon, and J.-Y. Pyun, "Signal processing for tracking of moving object in multi-impulse radar network system," Int. J. Distrib. Sen. Netw., vol. 2015, Jan. 2015

[105] B. Gulmezoglu, M. B. Guldogan, and S. Gezici, "Multiperson tracking with a network of ultrawideband radar sensors based on Gaussian mixture PHD filters," IEEE Sensors J., vol. 15, no. 4, pp. 2227-2237, Apr. 2015

[106] V.-H. Nguyen and J.-Y. Pyun, "Location detection and tracking of moving targets by a 2D IR-UWB radar system,' Sensors, vol. 15, no. 3, pp. 6740-6762, Mar. 2015.

[107] B. Sobhani, E. Paolini, M. Mazzotti, A. Giorgetti, and M. Chiani, "Multiple target tracking with particle filtering in UWB radar sensor networks," in Proc. Int. Conf. on Location and GNSS (ICL-GNSS), Gothenburg, Sweden, Jun. 2015, pp. 1-6.

[108] A. Eryildirim and M. B. Guldogan, "A Bernoulli filter for extended target tracking using random matrices in a UWB sensor network," IEEE Sensors J., vol. 16, no. 11, pp. 4362-4373, Jun. 2016.

[109] É. L. Souza, E. F. Nakamura, and R. W. Pazzi, "Target tracking for sensor networks: A survey," ACM Comput. Surv., vol. 49, no. 2, pp. 1-31, Jun. 2016

[110] D. Kocur, J. Fortes, and M. Švecová, "Multiple moving person tracking by UWB sensors: the effect of mutual shielding persons and methods reducing its impacts," EURASIP J. on Wireless Comm. and Netw., vol. 2017, no. 1, pp. 1-15, Apr. 2017.

[111] B. Sobhani, T. Zwick, and M. Chiani, "Target TOA association with the Hough transform in UWB radars," IEEE Trans. Aerosp. Electron. Syst., vol. 52, no. 2, pp. 743-754, Apr. 2016.

[112] S. Bartoletti, A. Conti, and A. Giorgetti, "Analysis of UWB radar sensor networks," in Proc. IEEE Int. Conf. on Commun. (ICC), Cape Town, SOUTH AFRICA, May 2010, pp. 1-6.

[113] Y. Yang, W. Yi, T. Zhang, G. Cui, L. Kong, and X. Yang, "Antenna placement of multistatic radar system with detection and localization performance," in Int. Conf. on Information Fusion (FUSION), Heidelberg, Germany, Jul. 2016, pp. 620-625

[114] G. Alirezaei, O. Taghizadeh, and R. Mathar, "Optimum power allocation in sensor networks for active radar applications," IEEE Trans. Wireless Commun., vol. 14, no. 5, pp. 2854-2867, May 2015.

[115] S. Haykin, "Cognitive radar: a way of the future," IEEE Signal Process. Mag., vol. 23, no. 1, pp. 30-40, Jan. 2006.

[116] Y. Nijsure, Y. Chen, S. Boussakta, C. Yuen, Y. H. Chew, and Z. Ding, "Novel system architecture and waveform design for cognitive radar radio networks," IEEE Trans. Veh. Technol., vol. 61, no. 8, pp. 3630 3642, Oct. 2012

[117] X. Li, Z. Hu, R. C. Qiu, Z. Wu, J. P. Browning, and M. C. Wicks, "Demonstration of cognitive radar for target localization under interference," IEEE Trans. Aerosp. Electron. Syst., vol. 50, no. 4, pp. 2440 2455 , Oct. 2014
[118] Y. Nijsure, G. Kaddoum, and H. Leung, "Cognitive chaotic UWBMIMO radar based on nonparametric bayesian technique," IEEE Trans. Aerosp. Electron. Syst., vol. 51, no. 3, pp. 2360-2378, Jul. 2015.

[119] J. D. Taylor, Ultra-wideband radar technology. CRC Press, 2000.

[120] A. Lazaro, D. Girbau, and R. Villarino, "Techniques for clutter suppression in the presence of body movements during the detection of respiratory activity through UWB radars," Sensors, vol. 14, no. 2, pp. 2595-2618, Feb. 2014.

[121] E. Piuzzi, S. Pisa, P. D’Atanasio, and A. Zambotti, "Radar cross section measurements of the human body for UWB radar applications," in IEEE Int. Instr. Meas. Tech. Conf., Graz, Austria, May 2012, pp. 12901293

[122] E. Piuzzi, P. D’Atanasio, S. Pisa, E. Pittella, and A. Zambotti, "Complex radar cross section measurements of the human body for breathactivity monitoring applications," IEEE Trans. Instrum. Meas., vol. 64, no. 8, pp. 2247-2258, Aug. 2015.

[123] A. Molisch, "Ultra-wide-band propagation channels," Proc. IEEE, vol. 97, no. 2, pp. 353-371, Feb. 2009.

[124] J. D. Taylor, Introduction to ultra wideband radar systems. CRC Press, 1995.

[125] D. Dardari, C. C. Chong, and M. Z. Win, "Threshold-based time-ofarrival estimators in UWB dense multipath channels," IEEE Trans. Commun., vol. 56, no. 8, pp. 1366-1378, Aug. 2008.

[126] I. Guvenc and Z. Sahinoglu, "Threshold-based TOA estimation for impulse radio UWB systems," in IEEE Int. Conf. Ultra-Wideband (ICUWB), Zurich, Switzerland, Sep. 2005, pp. 420-425.

[127] I. Guvenc, Z. Sahinoglu, P. Orlik, and H. Arslan, "Searchback algorithms for TOA estimation in non-coherent low-rate IR-UWB systems,' Wirel. Pers. Commun., vol. 48, pp. 585-603, Mar. 2009.

[128] S. Bartoletti, W. Dai, A. Conti, and M. Z. Win, "A mathematical model for wideband ranging," IEEE J. Sel. Topics Signal Process., vol. 9, no. 2, pp. 216-228, Mar. 2015.

[129] E. A. de Reyna, J. J. Murillo-Fuentes, and R. Boloix-Tortosa, "Blind low complexity time-of-arrival estimation algorithm for UWB signals," IEEE Signal Process. Lett., vol. 22, no. 11, pp. 2019-2023, Nov. 2015.

[130] J. Rovňáková and D. Kocur, "UWB radar signal processing for through wall tracking of multiple moving targets," in European Radar Conf. (EuRAD), Paris, France, Sep. 2010, pp. 372-375.

[131] D. Kocur, D. Novak, and J. Rovňáková, "Moving person tracking by UWB radar system in complex environment," in IEEE Int. Symp. on Intelligent Signal Proc., Funchal, Portugal, Sep. 2013, pp. 77-82.

[132] Y. He, P. Aubry, and F. L. Chevalier, "Ultra-wideband multistatic tracking of human targets," in IET Int. Radar Conf., Xi'an, China, Apr. 2013, pp. 1-5.

[133] Y. He, P. Aubry, F. L. Chevalier, and A. Yarovoy, "Decentralised tracking for human target in multistatic ultra-wideband radar," IET Radar, Sonar \& Navigation, vol. 8, no. 9, pp. 1215-1223, Dec. 2014.

[134] Y. He, "Human target tracking in multistatic ultra-wideband radar," Ph.D. dissertation, Delft University of Technology, Delft, Netherlands, Apr. 2014.

[135] H. Rohling, "Radar CFAR thresholding in clutter and multiple target situations," IEEE Trans. Aerosp. Electron. Syst., vol. AES-19, no. 4, pp. 608-621, Jul. 1983

[136] N. J. Willis, Bistatic Radar, 2nd ed. SciTech Publishing, Jan. 2007.

[137] M. Lops and M. Orsini, "Scan-by-scan averaging CFAR," IEE Proc. F - Radar and Signal Processing, vol. 136, no. 6, pp. 249-254, Dec. 1989.

[138] D. Urdzik and D. Kocur, "CFAR detectors for through wall tracking of moving targets by M-sequence UWB radar," in Int. Conf. Radioelektronika, Brno, Czech Republic, Apr. 2010, pp. 1-4.

[139] E. Conte, M. Lops, and G. Ricci, "Asymptotically optimum radar detection in compound-Gaussian clutter," IEEE Trans. Aerosp. Electron. Syst., vol. 31, no. 2, pp. 617-625, Apr. 1995.

[140] E. Conte, M. Longo, and M. Lops, "Analysis of the excision CFAR detector in the presence of fluctuating targets," IEE Proc. F - Radar and Signal Processing, vol. 136, no. 6, pp. 290-292, Dec. 1989.

[141] S. C. Johnson, "Hierarchical clustering schemes," Psychometrika, vol. 32, no. 3, pp. 241-254, 1967.

[142] C. D. Manning, P. Raghavan, and H. Schütze, Introduction to Information Retrieval. New York, NY, USA: Cambridge University Press, 2008.

[143] I. R. Goodman, R. P. S. Mahler, and H. T. Nguyen, Mathematics of Data Fusion. Kluwer Academic Publishers, 1997.

[144] R. P. S. Mahler, "“Statistics 102” for multisource-multitarget detection and tracking," IEEE J. Sel. Topics Signal Process., vol. 7, no. 4, pp. 376-389, Oct. 2013 
[145] _ "Multitarget Bayes filtering via first-order multitarget moments," IEEE Trans. Aerosp. Electron. Syst., vol. 39, no. 4, pp. 1152-1178, Oct. 2003.

[146] R. A. Singer, R. G. Sea, and K. B. Housewright, "Derivation and evaluation of improved tracking filters for use in dense multi-target environments," IEEE Trans. Inf. Theory, vol. 20, no. 4, pp. 423-432, Jul. 1974.

[147] Y. Bar-Shalom and E. Tse, "Tracking in a cluttered environment with probabilistic data association," Automatica, vol. 11, pp. 451-460, 1975.

[148] J. J. Stein and S. S. Blackman, "Generalized correlation of multi-target track data," IEEE Trans. Aerosp. Electron. Syst., vol. 11, no. 6, pp. 1207-1217, 1975.

[149] D. B. Reid, "An algorithm for tracking multiple targets," IEEE Trans. Autom. Control, vol. 24, no. 6, pp. 843-854, Dec. 1979.

[150] S. S. Blackman, "Multiple hypothesis tracking for multiple target tracking," IEEE Aerosp. Electron. Syst. Mag., vol. 19, no. 1, pp. 5854, Jan. 2004

[151] L. Lin, Y. Bar-Shalom, and T. Kirubarajan, "Track labeling and PHD filter for multi-target tracking," IEEE Trans. Aerosp. Electron. Syst., vol. 42, no. 3, Jul. 2006.

[152] K. Panta, B.-N. Vo, and S. Singh, "Novel data association schemes for the probability hypothesis density filter," IEEE Trans. Aerosp. Electron. Syst., vol. 43, no. 2, Apr. 2007

[153] K. Panta, D. E. Clark, and B.-N. Vo, "Data association and track management for the Gaussian mixture probability hypothesis density filter," IEEE Trans. Aerosp. Electron. Syst., vol. 45, no. 3, Jul. 2009.

[154] Time domain. [Online]. Available: http://www.timedomain.com

[155] Y. Wang, Q. Liu, and A. E. Fathy, "CW and pulse-Doppler radar processing based on FPGA for human sensing applications," IEEE Trans. Geosci. Remote Sens., vol. 51, no. 5, pp. 3097-3107, May 2013.

[156] M. Anabuki et al., "Ultrawideband radar imaging using adaptive array and Doppler separation," IEEE Trans. Aerosp. Electron. Syst., vol. 53, no. 1, pp. 190-200, Feb. 2017.

[157] R. P. S. Mahler, "PHD filters of higher order in target number," IEEE Trans. Aerosp. Electron. Syst., vol. 43, no. 4, pp. 1523-1543, Oct. 2007.

[158] B.-T. Vo, B.-N. Vo, and A. Cantoni, "The cardinality balanced multitarget multi-Bernoulli filter and its implementations," IEEE Trans. Signal Process., vol. 57, no. 2, pp. 409-423, Feb. 2009.

[159] B.-N. Vo, B.-T. Vo, N.-T. Pham, and D. Suter, "Joint detection and estimation of multiple objects from image observations," IEEE Trans. Signal Process., vol. 58, no. 10, pp. 5129-5141, Oct. 2010.

[160] B.-T. Vo, B.-N. Vo, R. Hoseinnezhad, and R. P. S. Mahler, "Robust multi-Bernoulli filtering," IEEE J. Sel. Topics Signal Process., vol. 7 , no. 3, pp. 399-409, Jun. 2013.

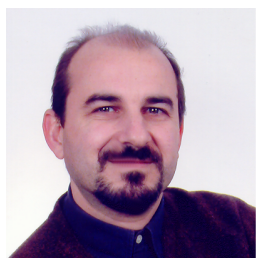

Marco Chiani (M'94-SM'02-F'11) received the Dr. Ing. degree (summa cum laude) in electronic engineering and the Ph.D. degree in electronic and computer engineering from the University of Bologna, Italy, in 1989 and 1993, respectively.

$\mathrm{He}$ is a Full Professor in Telecommunications at the University of Bologna. During summer 2001, he was a Visiting Scientist at AT\&T Research Laboratories, Middletown, NJ. Since 2003 he has been a frequent visitor at the Massachusetts Institute of Technology (MIT), Cambridge, where he presently holds a Research Affiliate appointment. His research interests are in the areas of communications theory, wireless systems, and statistical signal processing, including MIMO statistical analysis, codes on graphs, wireless multimedia, cognitive radio techniques, and ultra-wideband radios.

In 2012 he has been appointed Distinguished Visiting Fellow of the Royal Academy of Engineering, UK. He is the past chair (2002-2004) of the Radio Communications Committee of the IEEE Communication Society and past Editor of Wireless Communication (2000-2007) for the journal IEEE TRANSACTIONS ON COMmunications. He received the 2011 IEEE Communications Society Leonard G. Abraham Prize in the Field of Communications Systems, the 2012 IEEE Communications Society Fred W. Ellersick Prize, and the 2012 IEEE Communications Society Stephen O. Rice Prize in the Field of Communications Theory.

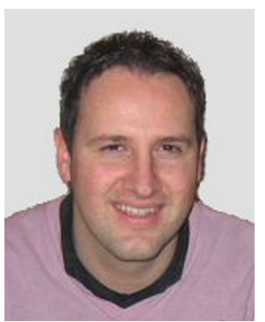

Andrea Giorgetti (S'98-M'04-SM'13) received the Dr. Ing. degree (summa cum laude) in electronic engineering and the Ph.D. degree in electronic engineering and computer science from the University of Bologna, Italy, in 1999 and 2003, respectively. From 2003 to 2005, he was a Researcher with the National Research Council, Italy. He joined the Department of Electrical, Electronic, and Information Engineering "Guglielmo Marconi," University of Bologna, as an Assistant Professor in 2006 and was promoted to Associate Professor in 2014. In spring 2006, he was with the Laboratory for Information and Decision Systems (LIDS), Massachusetts Institute of Technology (MIT), Cambridge, MA, USA. Since then, he has been a frequent visitor to the Wireless Information and Network Sciences Laboratory at the MIT, where he presently holds the Research Affiliate appointment.

His research interests include ultrawide bandwidth communication systems, active and passive localization, wireless sensor networks, and cognitive radio. He has co-authored the book Cognitive Radio Techniques: Spectrum Sensing, Interference Mitigation, and Localization (Artech House, 2012). He was the Technical Program Co-Chair of several symposia at the IEEE Int. Conf. on Commun. (ICC), and IEEE Global Commun. Conf. (Globecom). He has been an Editor for the IEEE COMMUNICATIONS LETTERS and for the IEEE TRansactions on Wireless Communications. He has been elected Chair of the IEEE Communications Society's Radio Communications Technical Committee.

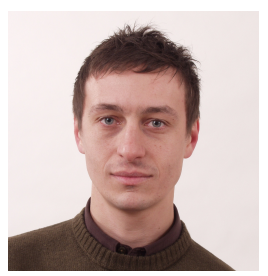

Enrico Paolini received the Dr. Ing. degree (summa cum laude) in Telecommunications Engineering in 2003 and the Ph.D. degree in Electrical Engineering in 2007, both from the University of Bologna, Italy. While working toward the Ph.D. degree, he was Visiting Research Scholar with the Department of Electrical Engineering of the University of Hawai'i at Manoa, Honolulu, HI, USA

From 2007 to 2010 , he held a postdoctoral position with the University of Bologna. He was a Visiting Scientist with the Institute of Communications and Navigation, German Aerospace Center, in 2012 and 2014, under DLR-DAAD fellowships. He joined the Department of Electrical, Electronic, and Information Engineering "Guglielmo Marconi", University of Bologna, as an Assistant Professor in 2010 and was promoted to Associate Professor in 2015. His research interests include channel coding, detection and tracking in radar systems, medium access protocols for a massive number of devices. He has contributed to EU projects FP-7 EUWB, FP-7 OPTIMIX, and FP-7 CONCERTO, and to several projects funded by the European Space Agency, including NEXCODE, HELIOS, SCAT.

Dr. Paolini is Editor of Coding and Information Theory for the IEEE TRANSACTIONS ON COMMUNICATIONS and past Editor (2012-2015) for the IEEE COMMUNICATIONS LETTERS. He served as co-chair of the ICC'14, ICC'15, and ICC'16 Workshop on Massive Uncoordinated Access Protocols (MASSAP), as chair of the 2018 IEEE European School of Information Theory (ESIT), and as treasurer of the 2011 IEEE International Conference on Ultra-Wideband (ICUWB). He is also serving as Technical Program Committee co-chair at IEEE GLOBECOM'19, Communication Theory Symposium. Dr. Paolini is member of the IEEE Communications Society and of the IEEE Information Theory Society and vice-chair of the ITSoc Italy Section Chapter. 